\title{
A Conversational Robot for Older Adults with Alzheimer's Disease
}

\author{
CHLOÉ POU-PROM, Li Ka Shing Knowledge Institute and University of Toronto, Canada \\ STEFANIA RAIMONDO, University of Toronto, Canada \\ FRANK RUDZICZ, Li Ka Shing Knowledge Institute, University of Toronto, and Vector Institute for \\ Artificial Intelligence, Canada
}

\begin{abstract}
Amid the rising cost of Alzheimer's disease (AD), assistive health technologies can reduce care-giving burden by aiding in assessment, monitoring, and therapy. This article presents a pilot study testing the feasibility and effect of a conversational robot in a cognitive assessment task with older adults with $\mathrm{AD}$. We examine the robot interactions through dialogue and miscommunication analysis, linguistic feature analysis, and the use of a qualitative analysis, in which we report key themes that were prevalent throughout the study. While conversations were typically better with human conversation partners (being longer, with greater engagement and less misunderstanding), we found that the robot was generally well liked by participants and that it was able to capture their interest in dialogue. Miscommunication due to issues of understanding and intelligibility did not seem to deter participants from their experience. Furthermore, in automatically extracting linguistic features, we examine how non-acoustic aspects of language change across participants with varying degrees of cognitive impairment, highlighting the robot's potential as a monitoring tool. This pilot study is an exploration of how conversational robots can be used to support individuals with $\mathrm{AD}$.
\end{abstract}

CCS Concepts: • Human-centered computing $\rightarrow$ Empirical studies in $\mathrm{HCI}$; Computer systems organization $\rightarrow$ Robotics;

Additional Key Words and Phrases: Assistive technology, dementia, human-robot interaction, dialogue

\section{ACM Reference format:}

Chloé Pou-Prom, Stefania Raimondo, and Frank Rudzicz. 2020. A Conversational Robot for Older Adults with Alzheimer's Disease. ACM Trans. Hum.-Robot Interact. 9, 3, Article 21 (May 2020), 25 pages.

https://doi.org/10.1145/3380785

\section{INTRODUCTION}

Alzheimer's disease (AD) is a neurodegenerative disease that primarily affects memory and leads to disorientation, loss of motivation, behavioural issues, and language problems [3, 4]. The decline

This work was funded by an Alzheimer Society of Canada Young Investigator grant (Rudzicz), a JP Bickell Foundation Research grant (Rudzicz), and an Ontario Ministry of Research, Innovation and Science Early Researcher Award (Rudzicz). Rudzicz is an inaugural CIFAR Chair in Artificial Intelligence.

Authors' addresses: C. Pou-Prom, Li Ka Shing Knowledge Institute, 2 Queen Street East, Toronto, Canada, University of Toronto, 714, Toronto, Ontario, M5C 3G7; email: Chloe.Pou-Prom@unityhealth.to; S. Raimondo, University of Toronto, 661 University Avenue, suite 710, Toronto, Ontario M5G 1M1; email: sraimond@cs.toronto.edu; F. Rudzicz, Li Ka Shing Knowledge Institute, 2 Queen Street East, Toronto, Canada, University of Toronto, 40 St. George Street, Toronto, Ontario M5S 2E4, and Vector Institute for Artificial Intelligenge, Toronto, Canada; email: frank@cs.toronto.edu.

Permission to make digital or hard copies of all or part of this work for personal or classroom use is granted without fee provided that copies are not made or distributed for profit or commercial advantage and that copies bear this notice and the full citation on the first page. Copyrights for components of this work owned by others than the author(s) must be honored. Abstracting with credit is permitted. To copy otherwise, or republish, to post on servers or to redistribute to lists, requires prior specific permission and/or a fee. Request permissions from permissions@acm.org.

(C) 2020 Copyright held by the owner/author(s). Publication rights licensed to ACM.

2573-9522/2020/05-ART21

https://doi.org/10.1145/3380785 
in language understanding and production can manifest in a variety of linguistic phenomena, including difficulty finding words, slower or more "empty" speech, and changes to the voice [11, 25]. Indeed, language can be a very sensitive measure for various cognitive and neuro-psychiatric conditions. Diagnosing dementia is typically done through assessments administered by a clinician, such as the Mini-Mental State Examination (MMSE) [9], which is nevertheless primarily used as an initial screening tool. Another popular assessment for detecting dementia is the "picture description" task, which requires individuals to describe the contents of an image and which is one of the more sensitive tests for detecting language disorders [10]. However, as the population ages, the prevalence of $\mathrm{AD}$ will increase drastically [15], prohibitively increasing the burden of assessment and monitoring.

The cost of care in $\mathrm{AD}$ tends to be expensive, and the burden on caregivers is rapidly increasing, along with the number of people with $\mathrm{AD}[6,12]$. Assistive health technologies, such as conversational robots, can help reduce the burden of assessment, monitoring, and therapy. For example, social robots have been used to provide personalized and affordable speech and cognitive therapy [39] and to engage individuals in social interactions, critical in the maintenance of good cognitive health [24,27]. Live-in robots may also provide more temporally fine-grained longitudinal analysis of speech and behaviour than is currently feasible for human health-care professionals. However, conversations with individuals with dementia are often subject to communication breakdowns and misunderstanding [33], which complicates the task for an artificial system. Also, speech recognition systems are typically not designed for individuals with language disabilities [47].

Here, we present Ludwig, a conversational robot for older adults with $\mathrm{AD}$ that administers the picture description task through speech. In this study, we investigate the nature of conversations between individuals with dementia and a robot and contrast them with conversations with another human. We focus on dialogue-level features, miscommunication, and perception of the robot, all of which are critical to assess the feasibility of using a robot for monitoring cognitive decline. We begin by recording conversations between adults diagnosed with $\mathrm{AD}$ and an experienced human (Hum). We then record the same individuals interacting with a robot in a "Wizard-of-Oz" (WoZ) setting, in which the robot has the benefit of the human-level interpretation of its operator. Finally, toward the goal of full autonomy, we extend our study with a third session, in which the participants interact with a simple autonomous implementation of a conversational robot (Rob). This allows us to investigate the effect of the gap in performance between the two robot conditions, and the effect of this on the quality of conversations. From audio recordings and transcripts of the three sessions (Hum vs. WoZ vs. Rob), we perform the type of linguistic analysis that would be performed in remote monitoring.

\section{RELATED WORK}

\subsection{Dementia and Conversation}

Language decline is one of the main symptoms of $\mathrm{AD}$. Acoustic, syntactic, and semantic features, including grammatical complexity and information content, can correlate strongly with cognitive decline [11,32]. Previous work has successfully assessed dementia based on automatic analysis of speech and text and has correlated the level of cognitive decline with speech impairment [45]. These correlations suggest that a conversational robot, which can collect large amounts of conversational data autonomously, could be useful in cognitive monitoring. The linguistic and acoustic analysis that we perform in this study is based on the analysis performed in the aforementioned studies.

Analysis of spontaneous connected speech offers a glimpse into semantic processing and syntactic complexity, and can be used to identify the deterioration of communication skills [29]. Often, 
the picture description task is used to elicit connected speech that then allows for the detection of subtle changes in cognitive decline, even in early stages of $\mathrm{AD}[10,13]$. Previous research has utilized machine learning approaches to assess dementia based on speech and transcripts of a picture description task $[8,19]$. Other language tasks, such as the naming task $[20]$, the verbal fluency task [18], and the story recall task [32], have also been used for automatic monitoring of AD.

Conversations with people with $\mathrm{AD}$ are far more likely to involve communication breakdown, which can be quantified through the trouble-source repair (TSR) framework [30]. TSR is a threepart methodology for identifying periods of confusion and consists of the trouble source, the repair initiator, and the resolution. Trouble sources can be phonological, morphological, semantic, or discourse related. A conversational agent should be able to identify trouble sources, or an equivalent paradigm, and perform the correct repair initiator or repair to maintain a conversation with individuals who might have more difficulty doing so.

\subsection{Robots and Dementia}

In early work, robotic companions were introduced to communities of people with dementia and imitated many of the benefits of animal therapy but without the burden or cost of maintaining live animals $[28,36]$. They showed positive effects, including increased brain activity and interaction, and decreased stress in both care receivers and caregivers [41,42] across multiple visits, suggesting that the positive emotional response is not merely due to the "novelty effect" [34].

A rationale for conversational abilities in companion robots arises given the improvements in cognition associated with increased social conversation [38]. As in human-human communication, conversations between older adults and care-giving robots ought to accommodate natural, free speech (as opposed to mere keyword interaction) and non-verbal cues (e.g., hand gestures, head pose, eye gaze).

One of the main challenges in developing a conversational robot is in building a robust automatic speech recognition (ASR) component. So far, ASR systems have performed poorly for individuals with dementia, and older adults generally, since they are typically designed for younger, healthy adults [47]. This may result in word-error-rates of $20-40 \%$ [31]. Fortunately, people tend to be forgiving of robots that cannot guarantee optimal performance, so long as the robot clearly acknowledges its own limitations [40].

In general, older adults are more likely to interact with a robot if it is more socially communicative [17]. Expressiveness is another important component-older adults prefer robots that are "less like robots" and mimic emotions [16]. For example, Sakai et al. found that participants were more interested in interacting with artificial conversational agents that produced social signals, such as smiles and nods [35].

Finally, Lee and Riek [23] proposed using the social model of aging as opposed to the deficit model of aging when building robot technologies for older adults. Viewing a robot as a "robot for successful aging" instead of an "assistive robot" can increase likeability and acceptability of that robot [23].

\section{METHODS}

Participants with AD performed the tablet-based picture description task across three sessions with (1) a human interlocutor, (2) a remotely controlled ("Wizard-of-Oz") robot, and (3) an autonomous robot. The first session provided a baseline for conversations surrounding a picture description. In contrast, the Session 2 Wizard-of-Oz condition demonstrated the effect of replacing the physical form of a human with that of a robot, while maintaining the conversational abilities of the human interlocutor. Finally, Session 3 explored the effect of also replacing the partner's conversational abilities with those of a simple autonomous agent. 
Table 1. Participant Demographics, Including Age, Sex, English-as-a-Second-Language (ESL), and MMSE Scores at the Times of Each Session

\begin{tabular}{|c|c|c|c|c|c|c|c|c|}
\hline ID & Age & Sex & ESL? & $\begin{array}{c}\text { MMSE } \\
\text { (Session 1) }\end{array}$ & $\begin{array}{c}\text { MMSE } \\
\text { (Session 2) }\end{array}$ & $\begin{array}{c}\text { Months/days } \\
\text { after Session } 1\end{array}$ & $\begin{array}{c}\text { MMSE } \\
\text { (Session 3) }\end{array}$ & $\begin{array}{c}\text { Months/days } \\
\text { after Session } 1\end{array}$ \\
\hline $\mathrm{P} 1$ & 94 & $\mathrm{~F}$ & & 13 & - & - & - & - \\
\hline $\mathrm{P} 2$ & 74 & $\mathrm{~F}$ & & 7 & 2 & $3.2 \mathrm{~m}$ & 0 & $11.6 \mathrm{~m}$ \\
\hline P3 & 88 & $\mathrm{~F}$ & & 19 & 17 & $3.3 \mathrm{~m}$ & 17 & $11.2 \mathrm{~m}$ \\
\hline $\mathrm{P} 4$ & 67 & M & & 2 & 1 & $3.2 \mathrm{~m}$ & 1 & $11.8 \mathrm{~m}$ \\
\hline P5 & 85 & $\mathrm{~F}$ & & 17 & 2 & $4.8 \mathrm{~m}$ & 0 & $11.6 \mathrm{~m}$ \\
\hline P6 & 93 & $\mathrm{~F}$ & & 1 & - & - & - & - \\
\hline P7 & 96 & $\mathrm{~F}$ & & 4 & - & - & - & - \\
\hline P8 & 92 & M & & 2 & 0 & $11.8 \mathrm{~m}$ & 0 & $12.2 \mathrm{~m}$ \\
\hline P9 & 93 & $\mathrm{~F}$ & $\checkmark$ & 11 & 13 & $3.3 \mathrm{~m}$ & 13 & $11.1 \mathrm{~m}$ \\
\hline P10 & 81 & $\mathrm{~F}$ & & 13 & 16 & $7.9 \mathrm{~m}$ & 16 & $8.4 \mathrm{~m}$ \\
\hline P11 & 93 & $\mathrm{~F}$ & & 7 & 7 & $7.9 \mathrm{~m}$ & 7 & $9.1 \mathrm{~m}$ \\
\hline P12 & 89 & $\mathrm{~F}$ & & 15 & 15 & $7 \mathrm{~d}$ & 15 & $35 \mathrm{~d}$ \\
\hline $\mathrm{P} 13$ & 92 & $\mathrm{~F}$ & $\checkmark$ & 12 & 12 & $7 \mathrm{~d}$ & 12 & $21 \mathrm{~d}$ \\
\hline P14 & 92 & $\mathrm{~F}$ & & 15 & 15 & $7 \mathrm{~d}$ & 15 & $21 \mathrm{~d}$ \\
\hline P15 & 83 & $\mathrm{~F}$ & & 8 & 8 & $7 \mathrm{~d}$ & 8 & $21 \mathrm{~d}$ \\
\hline P16 & 91 & $\mathrm{~F}$ & & 19 & 19 & $28 \mathrm{~d}$ & - & - \\
\hline P17 & 82 & M & & 11 & 11 & $14 \mathrm{~d}$ & 11 & $35 \mathrm{~d}$ \\
\hline P18 & 95 & $\mathrm{~F}$ & & 10 & 10 & $14 \mathrm{~d}$ & 10 & $28 \mathrm{~d}$ \\
\hline P19 & 91 & $\mathrm{~F}$ & & 21 & 21 & $14 \mathrm{~d}$ & - & - \\
\hline
\end{tabular}

A "-" indicates that a participant did not complete a session due to death or withdrawal.

The three recording sessions were collected over the span of a year. Each session consisted of a semi-structured conversation around the description of three different pictures, with previously unseen images in each session. In total, participants described 9 pictures ( 3 per session $\times 3$ sessions) with different conversation partners. Participants were seated at a table, with their conversation partner either seated across from them (Session 1) or standing on the table (Sessions 2 and 3). Participants viewed the pictures being discussed on the "Participant tablet." A similar "Operator tablet" also displayed the pictures and was used by either the human conversation partner (Session 1) or by the researcher (Sessions 2 and 3 ) to control the pictures appearing on the "Participant tablet" and to control the "Wizard-of-Oz" robot in Session 2.

\subsection{Participants}

We recruited 19 participants ( 3 male) diagnosed with AD from a nursing home. The participants had varying degrees of cognitive decline, with MMSE scores ranging from 2 to $19,{ }^{1}$ as reported in Table 1. All participants met the following inclusion criteria for participation: They were 55 years or older, they were able to speak and understand English at conversational levels, and they were able to hear normal levels of conversational speech. Exclusion criteria included a history of other forms of neurological illness and reported hearing or visual issues that would prevent participation.

To mitigate the effect of mood and speaker variability [44], we verified with nurses and personnel that participants were in an amenable condition before each session. Assent was provided by family members where informed consent was not possible, according to an approved ethics

\footnotetext{
$\overline{{ }^{1} \mathrm{~A} \text { score of } 18}$ to 23 is associated with mild cognitive impairment, 11 to 17 with moderate impairment, and 10 or less with severe cognitive impairment [9].
} 
(a)
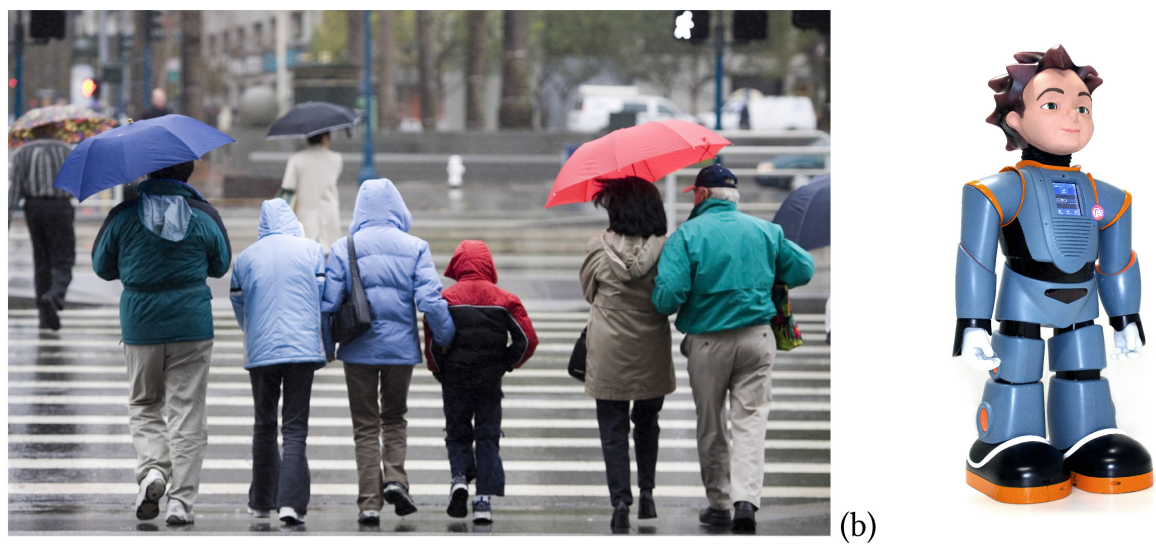

Fig. 1. Study materials. (a) Example image presented to participants. Each picture is annotated with physical entities, (e.g., "umbrella") from which we generate questions and prompts (e.g., "How many people do you see in the picture?"). Image courtesy Thomas Hawk (Flickr). (b) Ludwig the robot acted as the conversational partner in Session 2 and Session 3. Image courtesy Robokind. ${ }^{2}$

protocol approved by the University of Toronto (protocol 32947). There were only two exclusion criteria: Participants (1) must not have a history of other forms of neurological illness (to avoid other confounds) and (2) must not have reported hearing or visual issues that would prevent participation.

\subsection{Materials}

3.2.1 Stimuli. We selected 13 redistributable pictures from Flickr ${ }^{3}$ and from the Webber Photo Cards: Story Starters ${ }^{4}$ collection. A speech-language pathologist identified regions of interest and annotated these with physical entities, actions, and relations between entities, which formed a knowledge base for each image. From the knowledge base, we automatically generated a set of questions and prompts for each picture, to be used in the second session. Examples include specific questions (e.g., "Where is the truck?" and "What kind of truck is it?") and open-ended prompts (e.g., "What do you see in the picture?"). Figure 1 shows an example image.

3.2.2 Kinect Sensor. We placed a Kinect sensor across from the participants. The Kinect tracked six canonical facial feature animation units (AUs) at a sampling rate of $30 \mathrm{~Hz}$; i.e., lip raiser, jaw lowerer, lip stretcher, brow lowerer, lip corner depressor, and brow raiser. These AUs form a subset of the Candid3 model of human faces [2].

3.2.3 Ludwig the Robot. We used a Milo R25 robot from Robokind, ${ }^{5}$ as shown in Figure 1. The robot, which we named Ludwig, was about two feet tall and resembled a young friendly boy. It was able to perform a range of movements and had voice synthesis capabilities used in Sessions 2 and 3. The voice of the robot is the "Josh" US-English child voice from Acapela group. ${ }^{6}$

In the WoZ session, this robot was controlled by an "Operator"-Ludwig was able to wave, smile, and use its text-to-speech abilities to synthesize audio prompts. While the Operator was provided

\footnotetext{
${ }^{2}$ https://robots4autism.com/milo/.

${ }^{3}$ https://www.flickr.com/.

${ }^{4}$ https://www.superduperinc.com/products/view.aspx?pid=WFC134B\&s=webber-photo-cards-story-starters\#.

${ }^{5}$ https://robots4autism.com/.

${ }^{6}$ Hear a demo at https://www.acapela-group.com/voices/children-voices/.
} 


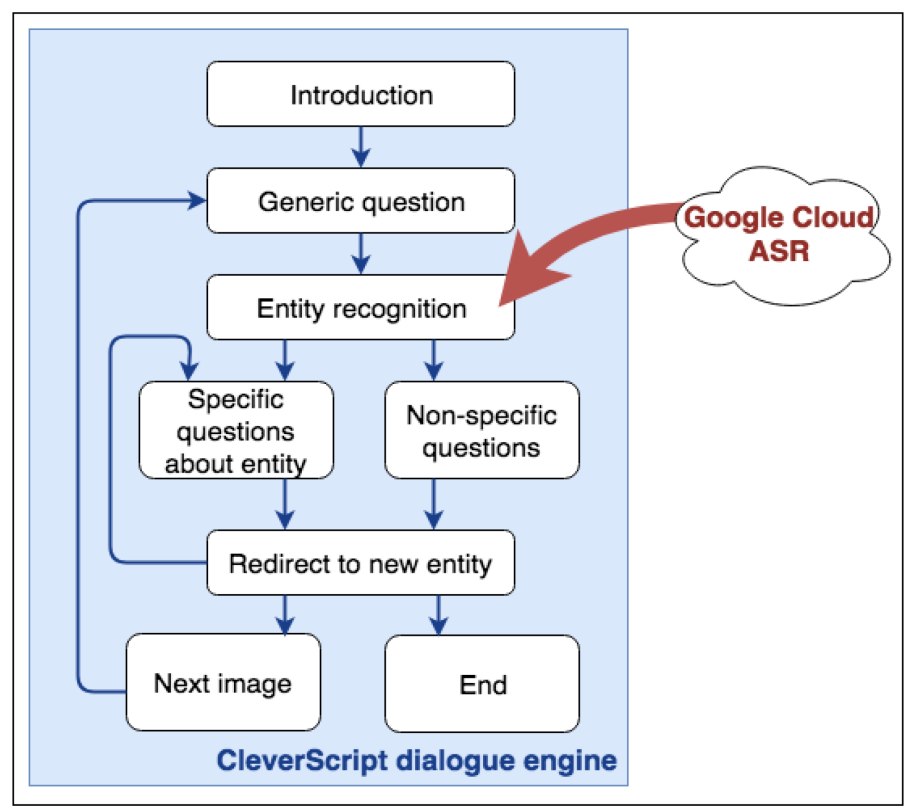

Fig. 2. Setup of the autonomous robot back-end. We used CleverScript for our dialogue engine and the Google Speech API for automatic speech recognition. We relied on the robot's internal voice synthesis for the text-to-speech component.

with a predefined list of questions for each image, they also had the ability to enter any other speech prompt.

In the third session, the robot was autonomous. We used a streaming version of Google's cloud automatic speech recognition $\mathrm{API}^{7}$ and CleverScript ${ }^{8}$ to build a rule-based dialogue engine. In post hoc analysis, we find word error rates at $49.72 \%$ for the third session, $76 \%$ of which was due to deletion errors, across all participants. We note that gold standard transcripts are used for subsequent analysis. Conversations followed the template described in Figure 2. After introducing itself, the robot would ask a generic question (e.g., "Can you tell me what you see in the picture?"). If the response matched a pre-defined entity (e.g., "umbrella," and "people"), then the dialogue system asked questions specific to the entity (e.g., "What colour is the umbrella?" and "Do the people know each other?"); otherwise, it asked non-specific or personal questions (e.g., "Do you think the picture is taken outside or inside?" and "Does the picture make you happy?"). After a given number of iterations, the conversation was re-directed to another image (e.g., "Let's look at another picture") or terminated (e.g., "Thanks for answering my questions!”).

\subsection{Data Collection}

In all sessions, we recorded audio and collected facial tracking data for the duration of the conversation. The robot's actions and utterances were also collected during Sessions 2 and 3.

We also administered usability questionnaires throughout the sessions (refer to Appendix A for more details). Prior to their first interaction with the robot, each participant completed a presession questionnaire about their familiarity with technology and with robots (e.g., "Have you ever

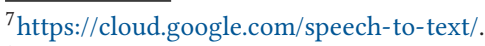

${ }^{8}$ https://www.cleverscript.com/.
} 
used a tablet before?" and "Are you nervous about interacting with a robot?"). Following the second and third sessions (i.e., the sessions with the "Wizard-of-Oz" and autonomous robot, respectively), participants completed post-session surveys, which focused on their interactions with the robot (e.g., "What did you like/dislike about the robot?" and "How did you feel when you were talking to the robot"). The questionnaires were semi-structured to allow for open-ended responses.

\subsection{Data Analysis}

Throughout our analysis, we perform comparisons across the following variables:

- Session - This refers to the picture description conversation sessions: Session 1 (human conversation partner), Session 2 (Wizard-of-Oz robot), and Session 3 (autonomous robot).

- Participant ID - The ID used to identify the participants. In some cases, we want to see if any differences are due to the participant.

- MMSE - We group each conversation into MMSE groups based on the participant's MMSE at the time the picture description session is conducted: Mild (score of 18 to 23), Moderate (score of 11 to 17), and Severe (score of 10 or less).

- Image Order - At each session, each participant describes three different pictures.

3.4.1 Language Analysis of Miscommunication and Dialogue. Audio of the conversations were transcribed at the utterance level and annotated by a trained speech-language pathologist with speaker information, dialogue acts (DAs), and trouble-source-repair (TSR) variables [30]. DAs are useful in conversational analysis and in developing conversational agents, because they encapsulate higher-level semantics of user "intentions." Pragmatics of this type have also themselves been used to assess for signs of dementia [1]. Trouble-source-repair variables are measures of communication breakdown and have also been used to analyze conversations of individuals with $\mathrm{AD}$ [30]. A subset of these annotations were also performed by two trained researchers, resulting in an inter-rater reliability (Fleiss' kappa) of 0.72 across all annotation categories.

The set of DAs, shown in Table 2, is the same used in the AMI Meeting Corpus, ${ }^{9}$ consisting of 14 acts tailored for a general, collaborative, task-oriented conversation. Note that the WoZ robot was capable of performing all DAs, while the autonomous robot in Session 3 was capable of only a subset (e.g., the autonomous robot did not perform any "Stall" or "Fragment" dialogue acts). The set of TSR variables is shown in Table 3.

To investigate whether DAs and TSRs vary significantly across our test conditions, for each annotated DA and TSR variable, we compute an $N$-way ANOVA on (i) the counts $(n)$ of utterances within a conversation and (ii) the percentage of utterances (\% utt) within a conversation. When comparing participant to partner speech, ANOVAs are computed to consider variation across Speaker, MMSE, Participant ID, and Image Order, considering all utterances together. When comparing participant or partner speech across conversation conditions, ANOVAs are computed to consider variation across Session, MMSE, Participant ID, and Image Order, separately for the participant and the conversational partner's utterances. We report variables that are significant at $\alpha<0.01$ and perform post hoc analysis using Bonferroni critical values to determine which covariates have significantly different means.

3.4.2 Linguistic Analysis for Cognitive Monitoring. As a proof of concept, and a demonstration of the kinds of linguistic analysis that could be performed on conversations with a robot, from conversation transcripts, we extract an extensive list of linguistic features using available

$\overline{{ }^{9} \text { http://groups.inf.ed.ac.uk/ami/corpus/. }}$ 
Table 2. Dialogue Acts (DA) from the AMI Meeting Corpus ${ }^{5}$ Dialogue Acts Encapsulate Higher-level Semantics of User "Intentions" and Are Useful in Conversational Analysis

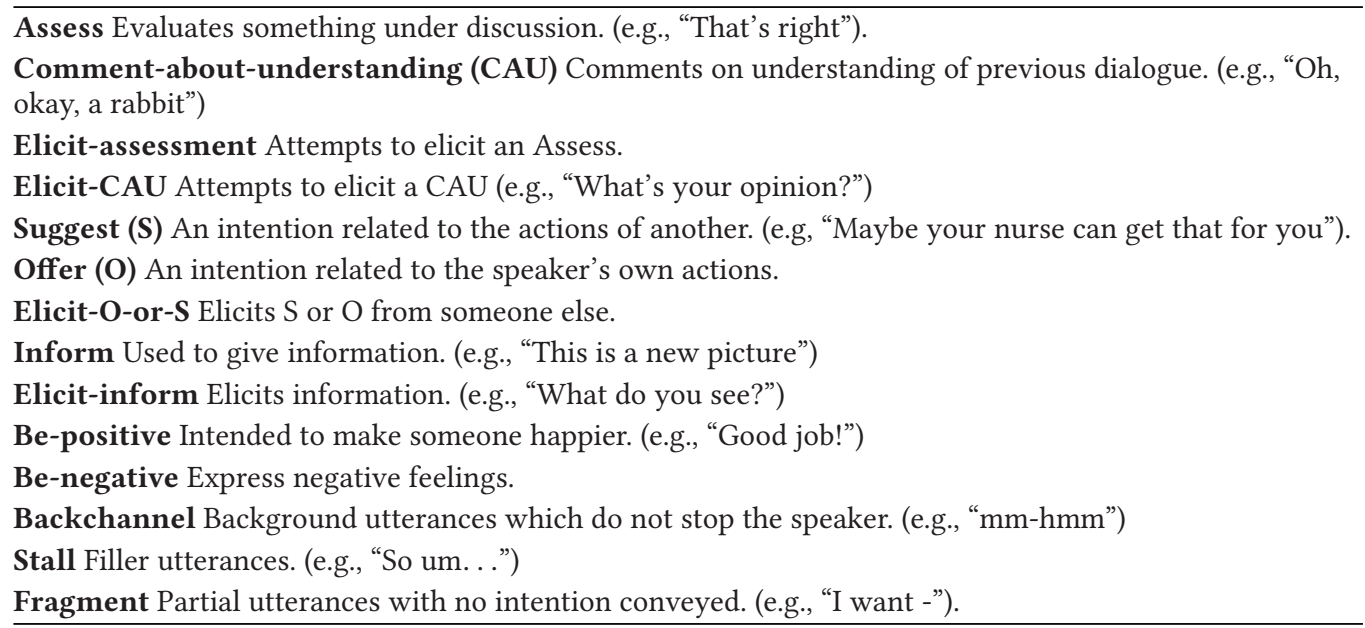

open-source software ${ }^{10}$ which have previously been used for analyzing cognition and speech impairment [21]. Note that we do not include acoustic features in the current analysis, since they would require segmentations of the audio at a per utterance level, which are unavailable.

We automatically extract lexical features related to the number of syllables and number of characters in words, the counts of dysfluencies (i.e., impairments to smooth, fluent speech), and measures of vocabulary richness (e.g., Honoré's statistic). Next, we compute syntactic features using the Stanford parser ${ }^{11}$ and compute ratios of context-free grammatical constructions, phrase type lengths, proportions and rates of parts-of-speech (e.g., conjunctions, pronouns, nouns), and density. We extract semantic features, including semantic cohesion, specificity, ambiguity, and similarity based on tree depth and number of senses from WordNet. Finally, we extract subjective measures such as crowd-sourced word norms for age-of-acquisition (i.e., typical ages at which a person learns each word) and valence, arousal, and dominance by computing the sentence-level mean values from the Bristol and Gihooly-Logie norms [14].

\section{RESULTS}

\subsection{Non-acoustic Language Analysis}

4.1.1 Partner and Participant. Table 4 presents the means and standard deviations of TSR and DA variables that differ significantly between the participant and their human partner in Session 1, as calculated by an ANOVA with Speaker (participant or partner), Participant ID, Image Order (the first, second, or third picture in a session), and MMSE as covariates.

Participants produced more Trouble Sources overall than their healthy human partners, with a larger proportion of turns having Discourse or Semantic Trouble Sources. Though the participants do not use more Repair Initiators overall than their partners, a higher proportion of the initiators they did use were of type L2-Non-specific terms. The human partner also performed more Repairs than the participant, with a higher percentage of Repetitions, Substitutions, and

\footnotetext{
${ }^{10}$ https://github.com/SPOClab-ca/COVFEFE.

${ }^{11}$ Version 2013-06-20, nlp.stanford.edu/software/lex-parser.shtml.
} 
Table 3. Trouble-Source Repair variables from Reference [30]

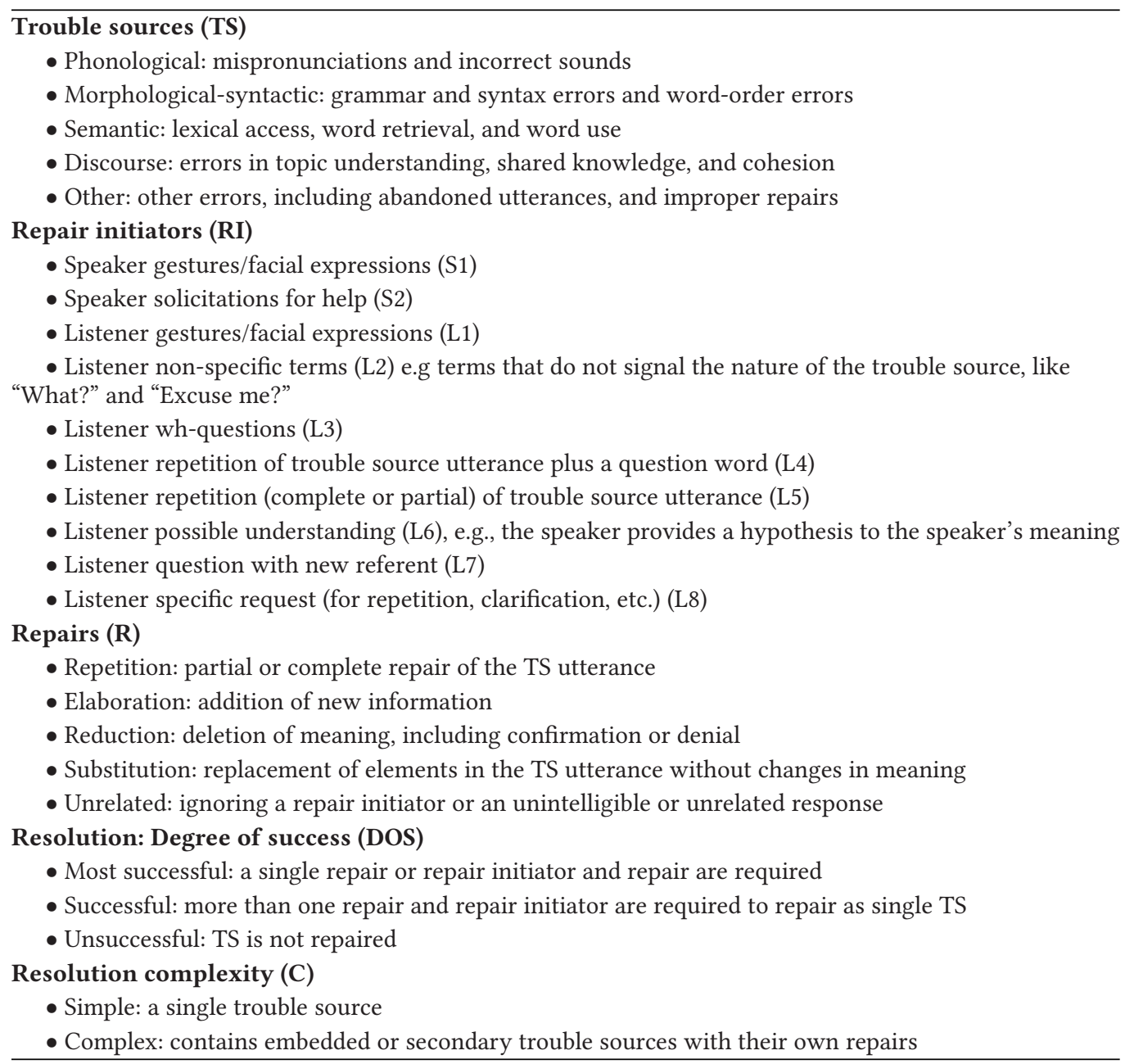

The Trouble-Source Repair framework is a three-part methodology for identifying periods of confusion. To maintain a conversation, a conversational agent should be able to identify trouble sources (or an equivalent paradigm) and respond appropriately (i.e., perform correct repair initiator or repair).

Elaborations. This corroborates similar findings by References [43] and [30], which overall suggest that conversational partners of individuals with AD carry the burden of conversational repair. We also observed that the participants used more Fragment DAs, which might further contribute to the production of their trouble sources. Stalls were used significantly more by the healthy human partner, which is unexpected but may relate to the relative low verbal rate of the participants. Stalls do not seem to contribute to increased trouble.

Differences in DAs mostly reflect the different roles of the participant and partner in the picture description task. Participants with dementia used more Inform acts, while the partner used more "Elicit Inform," "Elicit Assessment," and "Be Positive" utterances. However, the partner's increased use of "Elicit Comment" About Understanding appears to be another sign of managing the conversational repair process with the adult with dementia. 
Table 4. Language Differences between Human Partner and Participant in Session 1 (Human Conversation Partner) in Terms of Means and Standard Deviations of Number of Utterances of Specific Dialogue Acts and Trouble Source Repair Variables (see Tables 2 and 3 for Definitions), as well as Vocabulary and Utterance Lengths

\begin{tabular}{|c|c|c|c|c|c|c|}
\hline & \multicolumn{2}{|c|}{ Partner (Hum) } & \multicolumn{2}{|c|}{ Participant } & \\
\hline & & mean & std & mean & std & \\
\hline \multirow{14}{*}{ Dialogue Acts (DA) } & Be positive (\% utt) & 6.38 & 6.84 & 1.65 & 3.7 & $* * *$ \\
\hline & Be positive $(\mathrm{n})$ & 1.84 & 2.07 & 0.353 & 0.77 & $* * *$ \\
\hline & Elicit assessment (\% utt) & 14.8 & 9.09 & 1.05 & 2.45 & *** \\
\hline & Elicit assessment (n) & 4.32 & 3.22 & 0.353 & 0.955 & *** \\
\hline & Elicit CAU (n) & 2.52 & 2.36 & 0.0784 & 0.272 & *** \\
\hline & Elicit CAU (\% utt) & 9.91 & 9.52 & 0.17 & 0.621 & *** \\
\hline & Inform (\% utt) & 10.6 & 8.81 & 35.9 & 20.9 & *** \\
\hline & $\operatorname{Inform}(\mathrm{n})$ & 3.18 & 2.93 & 8.59 & 7.57 & *** \\
\hline & Elicit-inform (\% utt) & 19.7 & 13.6 & 4.55 & 7.24 & *** \\
\hline & Elicit-inform (n) & 5.45 & 4.32 & 1.14 & 1.56 & *** \\
\hline & Fragment (\% utt) & 5.44 & 7.07 & 23.9 & 22.3 & *** \\
\hline & Fragment (n) & 1.68 & 2.64 & 5.08 & 6.43 & ** \\
\hline & Stall (\% utt) & 17.5 & 11 & 7.96 & 9.85 & *** \\
\hline & Stall (n) & 5 & 3.92 & 2.39 & 3.52 & ** \\
\hline \multirow{4}{*}{ Trouble Sources (TS) } & TS (\% utt) & 20.5 & 15 & 33.4 & 24.4 & $* *$ \\
\hline & TS Discourse (\% utt) & 1.77 & 3.02 & 6.09 & 6.45 & $* * *$ \\
\hline & TS Discourse (n) & 0.571 & 0.951 & 1.29 & 1.35 & * \\
\hline & TS Semantic (\% utt) & 0.3 & 1.33 & 1.99 & 3.51 & * \\
\hline \multirow{4}{*}{ Repair Initiators (RI) } & RI: L2 (\% RI) & 11.8 & 23 & 31.5 & 39.5 & * \\
\hline & RI: L5 (\% RI) & 39.3 & 41.9 & 12.3 & 25.7 & $* * *$ \\
\hline & RI: L5 (\% utt) & 3.66 & 4.89 & 1.15 & 2.35 & * \\
\hline & RI: L5 (n) & 0.893 & 1 & 0.353 & 0.716 & * \\
\hline \multirow{11}{*}{ Repairs (R) } & R: Other (\% R) & 80 & 65.2 & 51.3 & 36.6 & * \\
\hline & R: Repetition (\% utt) & 3.31 & 4.13 & 1.19 & 2.27 & * \\
\hline & R: Repetition (n) & 1.18 & 1.69 & 0.275 & 0.493 & ** \\
\hline & $\mathrm{R}(\% \mathrm{utt})$ & 15.6 & 11.6 & 7.5 & 8.81 & ** \\
\hline & $\mathrm{R}(\mathrm{n})$ & 4.54 & 4.25 & 1.82 & 2.57 & ** \\
\hline & R: Elaboration (n) & 1.71 & 1.6 & 0.843 & 1.59 & * \\
\hline & R: Substitution (\% R) & 8.26 & 16.3 & 0 & 0 & ** \\
\hline & R: Substitution (\% utt) & 1.65 & 2.98 & 0 & 0 & ** \\
\hline & R: Substitution (n) & 0.5 & 0.915 & 0 & 0 & ** \\
\hline & Vocab. len. & 69.9 & 29.3 & 52.6 & 43.5 & * \\
\hline & Utterance len. (words) & 5.81 & 1.16 & 3.76 & 1.62 & *** \\
\hline
\end{tabular}

Values are provided in terms of number of utterances $(n)$, percentage of speaker utterances in a given conversation (\%utt), or percentage of utterances of a given class type (\%X, where $\mathrm{X}$ is the class, e.g., \%RI for percentage of Repair Initiations). Due to the large number of variables tested, only variables with differences are reported. ${ }^{* * *}$ denotes a $p$-value $<0.0001,{ }^{* *}<0.001,{ }^{*}<0.01$. 
Table 5. Participant Language across Session, in terms of Means and Standard Deviations of number of Utterances of Specific Dialogue Acts and Trouble Source Repair Variables (see Tables 2 and 3 for Definitions), as well as Vocabulary and Utterance Lengths

\begin{tabular}{llrrrrrrr}
\hline & & \multicolumn{1}{c}{ 1 (Hum) } & \multicolumn{2}{c}{ 2 (WoZ) } & \multicolumn{2}{c}{ 3 (Rob) } \\
\hline & & mean & std & mean & std & mean & std & \\
\hline & Assess (\% utt) & 25.7 & 18.7 & 19.7 & 23.6 & 15.9 & 21.6 & $*$ \\
& Assess (n) & 6.84 & 8.04 & 4.51 & 6.05 & 1.67 & 2.17 & $* * *$ \\
Dialogue Acts (DA) & Backchannel (\% utt) & 0.494 & 1.82 & 0 & 0 & 0 & 0 & $*$ \\
& Backchannel (n) & 0.161 & 0.565 & 0 & 0 & 0 & 0 & $*$ \\
& Fragment (\% utt) & 21.7 & 22.3 & 11 & 17.5 & 25.7 & 30.8 & $*$ \\
& Stall (n) & 2.18 & 3.42 & 1.98 & 3.71 & 0.769 & 1.22 & $*$ \\
\hline & TS (\% utt) & 30.5 & 25 & 15.7 & 21.3 & 36 & 32.6 & $* *$ \\
& TS (n) & 6.8 & 7.65 & 4 & 6.37 & 3.85 & 5 & $*$ \\
Trouble Sources (TS) & TS: Discourse (\% TS) & 23.2 & 28.1 & 0.981 & 5.98 & 2.56 & 11.8 & $* * *$ \\
& TS: Discourse (\% utt) & 5.54 & 6.4 & 0.138 & 0.653 & 0.892 & 4.95 & $* * *$ \\
& TS: Discourse (n) & 1.18 & 1.34 & 0.0667 & 0.33 & 0.128 & 0.656 & $* * *$ \\
& TS: Semantic (n) & 0.571 & 1.29 & 0.267 & 0.58 & 0.0769 & 0.27 & $*$ \\
\hline \multirow{2}{*}{ Repair Initiators (RI) } & RI: L8 (\% RI) & 1.02 & 6.73 & 0.855 & 5.73 & 6.72 & 16.8 & $*$ \\
& RI: L8 (\% utt) & 0.21 & 1.2 & 0.265 & 1.77 & 1.88 & 5.01 & $*$ \\
\hline \multirow{2}{*}{ Repairs (R) } & R (\% utt) & 6.83 & 8.68 & 2.14 & 3.56 & 2.33 & 5.46 & $*$ \\
& R: Repetition (n) & 0.25 & 0.477 & 0 & 0 & 0.0513 & 0.32 & $*$ \\
\hline & N. utt & 22.5 & 18.2 & 15.5 & 16.3 & 8.41 & 8.47 & $* * *$ \\
& Vocab. len. & 47.9 & 44.1 & 43.4 & 52.5 & 21.9 & 26.9 & $* * *$ \\
\hline
\end{tabular}

Values are provided in terms of number of utterances (n), percentage of speaker utterances in a given conversation (\%utt), or percentage of utterances of a given class type (\%X, where $\mathrm{X}$ is the class, e.g., \%RI for percentage of Repair Initiations). Due to the large number of variables tested, only variables with differences are reported. ${ }^{* * *}$ denotes a $p$-value $<0.0001$, ${ }^{* *}<0.001,{ }^{*}<0.01$.

4.1.2 Speaking with a Robot. Table 5 summarizes the TSR and DA variables that differed significantly in participant speech across sessions with the different conversational partners, as calculated by an ANOVA with Speaker, Image Order, Participant ID, and MMSE as covariates. Overall, participants were less talkative with the robot, with participant utterances declining from Sessions 1 to 3 , from an average of 21 to 13 to 4 per image and their vocabulary also declining in Session 3.

With the human partner, participants used significantly more "Assess" utterances, even when not prompted with more "Elicit Assessment" (see Table 5), suggesting perhaps more "openness" and willingness to offer opinions. According to Lai et al. [22], this may indicate increased conversation satisfaction and cohesiveness. Indeed, with the human partner, using more "Assess" and "Comment about Understanding" utterances than the robotic partner (see Table 6), the conversation appears to have been more collaborative.

Participants seemed to have had greater difficulty understanding the robotic partner. They used more Listener L8 (Specific Requests) repair initiators with the robot (see Table 5). In the longer conversations with the human partner, participants produced more "Discourse," or high-level, trouble sources. With the autonomous robot, compared to the more flexible "Wizard-of-Oz" robot, they produced more "Semantic" trouble sources overall.

While the participants responded differently to the robotic partners, the behaviour of the different partners-partly in response to participant changes-also differed. Table 6 summarizes the 
Table 6. Partner Language across Session, in Terms of Means and Standard Deviations of Number of Utterances of Specific Dialogue Acts and Trouble Source Repair Variables (see Tables 2 and 3 for Definitions), as Well as Vocabulary and Utterance Lengths

\begin{tabular}{|c|c|c|c|c|c|c|c|c|}
\hline & & \multicolumn{2}{|c|}{1 (Hum) } & \multicolumn{2}{|c|}{$2(\mathrm{WoZ})$} & \multicolumn{2}{|c|}{3 (Rob) } & \\
\hline & & mean & std & mean & std & mean & std & \\
\hline \multirow{9}{*}{ Dialogue Acts (DA) } & Assess $(\%$ utt $) \ddagger$ & 32.1 & 16.5 & 10.1 & 12.2 & 0 & 0 & $* * *$ \\
\hline & Backchannel (\% utt) $\ddagger$ & 0.639 & 2.03 & 0 & 0 & 0 & 0 & * \\
\hline & CAU (\% utt $) \ddagger$ & 7.02 & 6.61 & 0 & 0 & 0 & 0 & *** \\
\hline & Elicit-CAU (\% utt) $\ddagger$ & 9.91 & 9.52 & 12 & 14.4 & 3.82 & 6.34 & ** \\
\hline & $\operatorname{Inform}(\mathrm{n})$ & 3.18 & 2.93 & 0.644 & 1.15 & 0.667 & 1.01 & *** \\
\hline & Elicit-inform (\% utt) & 19.7 & 13.6 & 38.5 & 24.3 & 42.3 & 20.8 & $* * *$ \\
\hline & Be-positive (\% utt) & 6.38 & 6.84 & 12.1 & 12.8 & 14.1 & 13.8 & * \\
\hline & Fragment (\% utt) $\ddagger$ & 5.44 & 7.07 & 0.123 & 0.828 & 0.554 & 2.44 & *** \\
\hline & Stall (\% utt) $\ddagger$ & 17.5 & 11 & 3.03 & 7.01 & 0 & 0 & $* * *$ \\
\hline \multirow{4}{*}{ Trouble Sources (TS) } & TS (\% utt) & 20.5 & 15 & 37.8 & 24.9 & 46.9 & 26.9 & $* * *$ \\
\hline & TS: Phonological (\% TS) $\ddagger$ & 3.82 & 9.76 & 0.762 & 3.62 & 0 & 0 & * \\
\hline & TS: Morph.-Syn. (\% TS) $\neq$ & 6.66 & 11.8 & 0 & 0 & 2.01 & 7.22 & ** \\
\hline & TS: Other (\% utt) & 17.1 & 14.6 & 32.9 & 27.4 & 41.8 & 30.4 & *** \\
\hline \multirow{4}{*}{ Repair Initiators (RI) } & $\mathrm{RI}(\%$ utt $) \ddagger$ & 6.61 & 7.19 & 0 & 0 & 0 & 0 & $* * *$ \\
\hline & RI: L2 (n) & 0.339 & 0.668 & 0 & 0 & 0 & 0 & * \\
\hline & RI: L5 (\% RI $) \dagger \ddagger$ & 39.3 & 41.9 & 0 & 0 & 0 & 0 & $* * *$ \\
\hline & RI: L6 $(\% \mathrm{RI}) \dagger+$ & 6.46 & 20.6 & 0 & 0 & 0 & 0 & * \\
\hline \multirow{4}{*}{ Repairs (R) } & $\mathrm{R}(\%$ utt) & 15.6 & 11.6 & 25.5 & 20.8 & 31.1 & 24.5 & $* * *$ \\
\hline & R: Repetition $(\% \mathrm{R}) \ddagger$ & 20.8 & 26.5 & 27.4 & 37.1 & 5.64 & 19.3 & ** \\
\hline & R: Elaboration (\% utt) & 5.79 & 5.2 & 8.94 & 13.6 & 12.5 & 13.8 & * \\
\hline & R: Unrelated (\% utt $) \ddagger$ & 3.81 & 5.84 & 8.93 & 12.6 & 14.8 & 15 & $* * *$ \\
\hline \multirow[t]{2}{*}{ Repair Complexity } & Simple (\%utt) & 13.2 & 9.27 & 23.4 & 20.5 & 29.2 & 24.2 & $* * *$ \\
\hline & Most Successful (\% R) & 43 & 37.7 & 17.8 & 32.2 & 9.19 & 22 & $* * *$ \\
\hline \multirow[t]{5}{*}{ Resolution Success } & Successful $(\% \mathrm{R})$ & 8.07 & 13 & 4.1 & 11.9 & 0.855 & 5.34 & * \\
\hline & Unsuccessful (\%R) & 33.9 & 35.1 & 58.1 & 42.5 & 63.9 & 42.7 & *** \\
\hline & N. utt & 28.5 & 15.4 & 11.5 & 7.02 & 8.67 & 2.17 & $* * *$ \\
\hline & Len. utt. (sec) & 2.04 & 0.423 & 1.54 & 0.41 & 1.51 & 0.285 & $* * *$ \\
\hline & Vocab. len. & 69.9 & 29.3 & 29.5 & 12.7 & 28.4 & 6.95 & $* * *$ \\
\hline
\end{tabular}

Values are provided in terms of number of utterances $(\mathrm{n})$, percentage of speaker utterances in a given conversation (\%utt) or percentage of utterances of a given class type (\%X, where $\mathrm{X}$ is the class, e.g., \%RI for percentage of Repair Initiations). CAU refers to comments about understanding. Due to the large number of variables tested, only variables with differences are reported. ${ }^{* * *}$ denotes a $p$-value $<0.0001,{ }^{* *}<0.001,{ }^{*}<0.01$.

$\dagger$ Also significant for $\%$ utterances.

$\ddagger$ Also significant for number of total utterances $(\mathrm{n})$.

TSR and DA variables that differed significantly in partner speech across sessions. In our implementation, the robotic partner was less able to participate in the repair process. Although the robotic partner still used more Elaboration and Repetition repairs than the participant, compared to the Wizard-of-Oz and human partners, a large proportion of the autonomous robot's repairs were "Unrelated" (or unhelpful). Furthermore, the human partner had significantly more "Most Successful" repairs, but the majority of the robot's repairs were "Unsuccessful" (see Table 6). For example, 
Table 7. Participant Language Use across MMSE, in Terms of Means and Standard Deviations of Number of Utterances of Specific Dialogue Acts and Trouble Source Repair Variables (see Tables 2 and 3 for Definitions), as Well as Vocabulary and Utterance Lengths

\begin{tabular}{llrrrrrrr}
\hline & & \multicolumn{2}{c}{ mild } & \multicolumn{2}{c}{ moderate } & \multicolumn{2}{c}{ severe } \\
& & \multicolumn{2}{c}{ (MMSE 21-25) } & \multicolumn{2}{c}{ (MMSE 11-20) } & \multicolumn{2}{c}{ (MMSE $\leq 10)$} & \\
\hline & & mean & std & mean & std & mean & std & \\
\hline \multirow{2}{*}{ Dialogue Acts (DA) } & CAU (\% utt) & 7.83 & 7.6 & 11.2 & 16.3 & 3.94 & 7.52 & $* * *$ \\
& CAU (n) & 1.8 & 2.18 & 2.3 & 2.78 & 0.787 & 1.59 & $* * *$ \\
\hline \multirow{3}{*}{ Repair Initiators (RI) } & RI (\% utt) & 8.29 & 8.05 & 11.9 & 17.3 & 4 & 8.45 & $*$ \\
& RI (n) & 1.93 & 2.28 & 2.47 & 3.15 & 0.738 & 1.45 & $* * *$ \\
& RI: L2 (\% utt) & 2.7 & 3.22 & 3.68 & 6.98 & 2.3 & 5.83 & $* * *$ \\
& RI: L2 (n) & 0.6 & 0.737 & 0.875 & 1.55 & 0.344 & 0.772 & $* * *$ \\
\hline
\end{tabular}

Values are provided in terms of number of utterances (n), percentage of speaker utterances in a given conversation (\%utt), or percentage of utterances of a given class type (\%X, where X is the class, e.g., \%RI for percentage of Repair Initiations). CAU refers to comments about understanding. Due to the large number of variables tested, only variables with differences are reported. ${ }^{* * *}$ denotes a $p$-value $<0.0001,{ }^{* *}<0.001,{ }^{*}<0.01$.

Robot: Is it raining in the picture?

P18: $\quad$ mm... oh

Robot: How many umbrellas do you see in the picture?

P18: Yes good.

Similarly, the robot, in either condition, with utterances dominated by Elicit-inform DAs, initiated far fewer repairs and produced more "Discourse," "Other," and overall trouble sources than the human partner (see Table 5).

4.1.3 Across MMSE. Participants differed significantly in DA "Comment About Understanding" across MMSE (see Table 7), with participants with severe dementia using far fewer. Coupled with a decrease in repair initiators, specifically with Listener L2 (Non-specific terms), this might suggest that participants with severely low MMSE (i.e., $\leq 10$ ) are less aware of their lack of understanding.

We compare the amount of speech produced across sessions and across participants grouped by the level of cognitive impairment in Table 8. People in the moderate MMSE group speak more, on average, than people with severe MMSE, which is consistent with our expectation. The mild group is under-powered statistically in this analysis to draw a conclusion about that group.

Partners adjusted their language to use more "Assess" utterances with participants with severely low MMSE (see Table 9), sometimes answering their own questions or providing more direct feedback to these participants. They also used fewer Listener L2 (non-specific terms) repair initiators and fewer "Substitution" repairs with people with severely low MMSE.

4.1.4 Across Participant. There are significant differences across all categories of annotated variables and across conversations with different participants, suggesting that it is important to adapt to individual participants rather than generalize.

4.1.5 Summary. We analyzed the transcripts of picture description sessions using annotations of dialogue acts and trouble-source-repair variables (as described in Tables 2 and 3). We first looked at differences between participants and their human conversation partners, and we observed that conversation partners were tasked with carrying the burden of conversational repair, as exemplified by their increased use of repair variables (e.g., repetitions). Next, we compared participant interactions across the different sessions and found that participants seemed to be more 
Table 8. Number of Words and Time Spent Speaking across Sessions, and Levels of Cognitive Impairment, Averaged across Members of Each Group

\begin{tabular}{c|rrr}
\hline Session & \multicolumn{1}{|c}{ Mild } & Moderate & \multicolumn{1}{c}{ Severe } \\
\hline \multirow{2}{*}{1} & 126.0 words & 350.2 words & 258.1 words \\
& $72.8 \mathrm{~s}$ & $156.0 \mathrm{~s}$ & $143.8 \mathrm{~s}$ \\
\hline \multirow{2}{*}{2} & 6.0 words & 405.0 words & 145.0 words \\
& $17.1 \mathrm{~s}$ & $194.6 \mathrm{~s}$ & $98.4 \mathrm{~s}$ \\
\hline \multirow{2}{*}{3} & N/A & 183.3 words & 50.4 words \\
& N/A & $106.6 \mathrm{~s}$ & $37.4 \mathrm{~s}$ \\
\hline
\end{tabular}

No participants with mild cognitive impairment completed the third session.

Table 9. Partner Language across MMSE, in Terms of Means and Standard Deviations of Number of Utterances of Specific Dialogue Acts and Trouble Source Repair Variables (see Tables 2 and 3 for

Definitions), as Well as Vocabulary and Utterance Lengths

\begin{tabular}{|c|c|c|c|c|c|c|c|c|}
\hline & & \multicolumn{2}{|c|}{$\begin{array}{c}\text { mild } \\
(\mathrm{MMSE} 21-25) \\
\end{array}$} & \multicolumn{2}{|c|}{$\begin{array}{c}\text { moderate } \\
(\mathrm{MMSE} 11-20) \\
\end{array}$} & \multicolumn{2}{|c|}{$\begin{array}{c}\text { severe } \\
(\mathrm{MMSE} \leq 10)\end{array}$} & \\
\hline & & mean & std & mean & std & mean & std & \\
\hline Dialogue Acts (DA) & Assess (\% utt) & 33.1 & 19.4 & 15.2 & 18.9 & 12.8 & 15.7 & * \\
\hline Repair Initiators (RI) & RI: L2 (\% RI) & 3.33 & 8.8 & 6.25 & 19.4 & 3.42 & 12.1 & ** \\
\hline \multirow{3}{*}{ Repairs (R) } & Repairs (n) & 3.93 & 2.05 & 3.58 & 3.8 & 3.33 & 3.1 & $* * *$ \\
\hline & R: Substitution (\% utt) & 0.951 & 2.02 & 1.32 & 3.22 & 1.01 & 2.78 & *** \\
\hline & R: Substitution (n) & 0.2 & 0.414 & 0.344 & 0.84 & 0.18 & 0.428 & $* * *$ \\
\hline
\end{tabular}

Values are provided in terms of number of utterances (n), percentage of speaker utterances in a given conversation (\%utt), or percentage of utterances of a given class type (\%X, where $\mathrm{X}$ is the class, e.g., \%RI for percentage of Repair Initiations). Due to the large number of variables tested, only variables with differences are reported. ${ }^{* * *}$ denotes a p-value $<0.0001$, ${ }^{* *}<0.001,{ }^{*}<0.01$.

collaborative with a human as a conversation partner than with the robot. Both human and the Wizard-of-Oz robot were able to perform repairs, but the human (i.e., in Session 1) had more instances of successful repairs. Finally, we analyzed the DAs and TSRs across MMSE groups (i.e., Mild vs. Moderate vs. Severe), examining how participant and partner non-acoustic aspects of language differed according to the participant's MMSE. We find that the conversation partner adjusted aspects of their language for the different groups, especially for participants in the severe MMSE group.

\subsection{Linguistic Analysis for Cognitive Monitoring}

We extract linguistic features, as described in Section 3.4.2, and further analyze them to assess the feasibility of using the robot to monitor cognitive impairment. For each linguistic feature, we perform $N$-way ANOVAs with MMSE, Session, Participant ID, and Image Order as covariates. We report the $F$ measure and $p$-value of the top 15 features (for readability) based on the $p$-values of the MMSE covariate in Table 10. Appendix B describes details on the features.

Both average PP (prepositional phrase) length and average VP (verb phrase) length, corresponding to the lengths of prepositional and verb phrases, respectively, significantly differentiate between MMSE groups. We find that the proportion of PP phrases with respect to the total number of grammatical constituents is also significant. The moving average type-token-ratio measures vocabulary richness and is the ratio obtained by dividing the types (i.e., the total number of unique words) 
Table 10. $n$-way ANOVA on Linguistic Features with Session, MMSE, Participant, and Image Order as Covariates

\begin{tabular}{lcccr}
\hline & \multicolumn{1}{c}{ Session } & \multicolumn{1}{c}{ MMSE } & Participant & Image Order \\
\hline Average PP length & $4.04(0.0201)^{*}$ & $51.5(1.08 \mathrm{e}-16)^{* * *}$ & $8.21(1.39 \mathrm{e}-13)^{* * *}$ & $1.29(0.278)$ \\
Min. WordNet depth & $12.7(1.00 \mathrm{e}-5)^{* * *}$ & $50.1(2.24 \mathrm{e}-16)^{* * *}$ & $9.67(9.73 \mathrm{e}-16)^{* * *}$ & $1.20(0.305)$ \\
(SD) - nouns & & & & \\
Max. WordNet depth & $10.6(5.92 \mathrm{e}-5)^{* * *}$ & $45.7(2.46 \mathrm{e}-15)^{* * *}$ & $8.06(2.33 \mathrm{e}-13)^{* * *}$ & $1.30(0.277)$ \\
(SD) - nouns & & & & \\
Average VP length & $7.07(1.27 \mathrm{e}-3)^{* *}$ & $45.6(2.62 \mathrm{e}-15)^{* * *}$ & $11.6(2.78 \mathrm{e}-18)^{* * *}$ & $0.442(0.644)$ \\
Word length & $3.51(0.0331)^{* *}$ & $44.1(5.93 \mathrm{e}-15)^{* * *}$ & $8.43(6.49 \mathrm{e}-14)^{* * *}$ & $0.514(0.600)$ \\
Age-of-acquisition - & $8.64(3.19 \mathrm{e}-4)^{* * *}$ & $38.9(1.24 \mathrm{e}-13)^{* * *}$ & $5.30(9.60 \mathrm{e}-9)^{* * *}$ & $0.561(0.572)$ \\
nouns & & & & \\
MATTR (40) & $21.1(1.61 \mathrm{e}-8)^{* * *}$ & $38.7(1.42 \mathrm{e}-13)^{* * *}$ & $9.28(3.59 \mathrm{e}-15)^{* * *}$ & $0.600(0.550)$ \\
MATTR (20) & $22.5(5.48 \mathrm{e}-9)^{* * *}$ & $38.0(2.17 \mathrm{e}-13)^{* * *}$ & $8.50(5.04 \mathrm{e}-14)^{* * *}$ & $0.836(0.436)$ \\
Familiarity - nouns & $11.8(2.09 \mathrm{e}-5)^{* * *}$ & $36.9(4.16 \mathrm{e}-13)^{* * *}$ & $5.93(7.20 \mathrm{e}-10)^{* * *}$ & $1.59(0.208)$ \\
MATTR (30) & $21.0(1.65 \mathrm{e}-8)^{* * *}$ & $36.8(4.52 \mathrm{e}-13)^{* * *}$ & $8.18(1.55 \mathrm{e}-13)^{* * *}$ & $0.552(0.577)$ \\
Function words & $2.09(0.128)$ & $36.2(6.49 \mathrm{e}-13)^{* * *}$ & $4.31(6.18 \mathrm{e}-7)^{* * *}$ & $1.45(0.239)$ \\
Mean length of & $4.00(0.0209){ }^{*}$ & $36.0(7.41 \mathrm{e}-13)^{* * *}$ & $10.1(2.34 \mathrm{e}-16)^{* * *}$ & $0.331(0.719)$ \\
sentence & & & & \\
PP type proportion & $1.11(0.333)$ & $35.9(7.71 \mathrm{e}-13)^{* * *}$ & $6.58(5.73 \mathrm{e}-11)^{* * *}$ & $1.82(0.167)$ \\
MATTR (50) & $18.9(7.78 \mathrm{e}-8)^{* * *}$ & $35.3(1.11 \mathrm{e}-12)^{* * *}$ & $9.48(1.83 \mathrm{e}-15)^{* * *}$ & $2.05(0.133)$ \\
WordNet ambiguity & $10.7(5.60 \mathrm{e}-5)^{* * *}$ & $34.9(1.39 \mathrm{e}-12)^{* * *}$ & $8.27(1.13 \mathrm{e}-13)^{* * *}$ & $1.54(0.218)$ \\
(SD) - nouns & & & & \\
\hline
\end{tabular}

We report the $F$ measure and $p$-value (in parentheses). ${ }^{* * *}$ denotes a $p$-value $<0.001,{ }^{* *}<0.01,{ }^{*}<0.05$.

occurring in a text or utterance by its tokens (i.e., the total number of word instances), computed over a moving window of size $k$ over the transcript, and then averaged over all windows [7]. In this case, we set $k$ to $20,30,40$, and 50. Word length (in characters) and mean length of sentence (in words) also significantly differentiate between MMSE groups.

WordNet depth of nouns is extracted from a lexical database that groups English words into sets of synonyms [26]. These sets are organized in a hierarchical manner from more general to more specific, so the notion of depth is a proxy for the specificity of word. We find that the standard deviations of the minimum and maximum depths for each noun in the transcript are significant across groups. Each noun is given one or many definitions, and we use the total number of senses to define ambiguity.

We use the Bristol [37] and Gilhoolie-Logie [14] ratings to compute the mean values of age-ofacquisition (i.e., the age at which a person learned a word) and familiarity (i.e., how often a word is used, seen, or heard). From tagging our transcripts for parts-of-speech, we obtain the counts of function words (i.e., words that signal grammatical relationships in a sentence).

We perform Tukey's honest significant difference (HSD) test as a post hoc analysis to find which means within the MMSE groups and which means within the Session groups differ from each other. We report results of the Tukey HSD test in Sections 4.2.1 and 4.2.2.

4.2.1 Linguistic Features across MMSE. Results of the Tukey HSD test on MMSE are reported in Table 11. We find that the greatest differences in features are between the Severe and Mild groups and the Severe and Moderate group.

4.2.2 Linguistic Features across Sessions. Results of the Tukey HSD test on Session are reported in Table 12. Most differences in features are between Session 1 and Session 3. For example, nouns 
Table 11. Post Hoc Analysis of the ANOVA on MMSE (Mild vs. Moderate vs. Severe) Showing Differences between Group Means

\begin{tabular}{lrrr}
\hline & Moderate -Mild & \multicolumn{1}{c}{ Severe -Mild } & Severe - Moderate \\
\hline Average PP length & $0.586(0.142)$ & $-1.34(8.65 \mathrm{e}-5)^{* * *}$ & $-1.93(2.54 \mathrm{e}-14)^{* * *}$ \\
Min. WordNet depth (SD) - nouns & $-0.0677(0.923)$ & $-1.11(2.66 \mathrm{e}-8)^{* * *}$ & $-1.04(2.82 \mathrm{e}-14)^{* * *}$ \\
Max. WordNet depth (SD) - nouns & $-0.0131(0.998)$ & $-1.14(2.90 \mathrm{e}-7)^{* * *}$ & $-1.13(3.86 \mathrm{e}-14)^{* * *}$ \\
Average VP length & $0.916(0.0138)$ & $-0.990(7.29 \mathrm{e}-3)^{* *}$ & $-1.91(2.80 \mathrm{e}-14)^{* * *}$ \\
Word length & $-0.121(0.815)$ & $-1.21(6.44 \mathrm{e}-8)^{* * *}$ & $-1.08(1.03 \mathrm{e}-13)^{* * *}$ \\
Age-of-acquisition - nouns & $18.0(0.769)$ & $-121(2.80 \mathrm{e}-5)^{* * *}$ & $-139(1.86 \mathrm{e}-13)^{* * *}$ \\
MATTR (40) & $0.0836(0.340)$ & $-0.238(3.36 \mathrm{e}-4)^{* * *}$ & $-0.322(1.14 \mathrm{e}-13)^{* * *}$ \\
MATTR (20) & $0.0447(0.765)$ & $-0.294(3.70 \mathrm{e}-5)^{* * *}$ & $-0.339(2.99 \mathrm{e}-13)^{* * *}$ \\
Familiarity - nouns & $29.2(0.858)$ & $-258(2.79 \mathrm{e}-5)^{* * *}$ & $-287(6.59 \mathrm{e}-13)^{* * *}$ \\
MATTR (30) & $0.0679(0.527)$ & $-0.262(1.86 \mathrm{e}-4)^{* * *}$ & $-0.330(3.84 \mathrm{e}-13)^{* * *}$ \\
Function words & $2.62 \mathrm{e}-3(0.994)$ & $-0.130(8.04 \mathrm{e}-6)^{* * *}$ & $-0.132(1.84 \mathrm{e}-12)^{* * *}$ \\
Mean length of sentence & $1.50(2.84 \mathrm{e}-3){ }^{* * *}$ & $-0.836(0.152)$ & $-2.34(3.75 \mathrm{e}-13)^{* * *}$ \\
PP type proportion & $-4.09 \mathrm{e}-4(1.00)$ & $-0.0972(5.78 \mathrm{e}-6)^{* * *}$ & $-0.0968(2.63 \mathrm{e}-12)^{* * *}$ \\
MATTR (50) & $0.107(0.147)$ & $-0.189(3.47 \mathrm{e}-3)^{* *}$ & $-0.296(5.00 \mathrm{e}-13)^{* * *}$ \\
WordNet ambiguity - nouns & $0.348(0.643)$ & $-1.63(1.65 \mathrm{e}-4)^{* * *}$ & $-1.98(1.31 \mathrm{e}-12)^{* * *}$ \\
\hline
\end{tabular}

We report the difference in means and $p$-values (in parentheses) following a Tukey honest significant difference test with a $95 \%$ familywise confidence level. ${ }^{* * *}$ denotes a $p$-value $<0.001,{ }^{* *}<0.01,{ }^{*}<0.05$.

Table 12. Post Hoc Analysis of the ANOVA for Linguistic Features on Session (Session 1 vs. Session 2 vs. Session 3)

\begin{tabular}{lrrr}
\hline & Session 2 - Session 1 & Session 3 - Session 1 & Session 3 - Session 2 \\
\hline Average PP length & $-0.358(0.223)$ & $-0.625(0.0166)^{*}$ & $-0.268(0.491)$ \\
Min. WordNet depth (SD) - nouns & $-0.343(0.0183)^{*}$ & $-0.647(6.14 \mathrm{e}-6)^{* * *}$ & $-0.304(0.0684)$ \\
Max. WordNet depth (SD) - nouns & $-0.364(0.0273)^{*}$ & $-0.661(4.02 \mathrm{e}-5)^{* * *}$ & $-0.296(0.131)$ \\
Average VP length & $-0.594(0.0240)^{*}$ & $-0.824(1.67 \mathrm{e}-3)^{* *}$ & $-0.229(0.617)$ \\
Word length & $-0.321(0.0589)$ & $-0.314(0.0814)$ & $6.86 \mathrm{e}-3(0.999)$ \\
Age-of-acquisition - nouns & $-34.8(0.139)$ & $-78.6(1.85 \mathrm{e}-4)^{* * *}$ & $-43.8(0.0740)$ \\
MATTR (40) & $-0.112(0.0215)^{*}$ & $-0.280(6.79 \mathrm{e}-9)^{* * *}$ & $-0.168(9.16 \mathrm{e}-4)^{* * *}$ \\
MATTR (20) & $-0.153(2.50 \mathrm{e}-3)^{* *}$ & $-0.312(2.59 \mathrm{e}-9)^{* * *}$ & $-0.159(4.32 \mathrm{e}-3)^{* *}$ \\
Familiarity - nouns & $-114(0.0108)^{*}$ & $-191(1.66 \mathrm{e}-5)^{* * *}$ & $-77.8(0.159)$ \\
MATTR (30) & $-0.137(6.19 \mathrm{e}-3)^{* *}$ & $-0.295(7.38 \mathrm{e}-9)^{* * *}$ & $-0.158(3.62 \mathrm{e}-3)^{* *}$ \\
Function words & $-0.0320(0.190)$ & $-0.0324(0.209)$ & $-3.45 \mathrm{e}-4(1.00)$ \\
Mean length of sentence & $-0.610(0.126)$ & $-0.867(0.0228)^{*}$ & $-0.257(0.730)$ \\
PP type proportion & $6.88 \mathrm{e}-4(0.999)$ & $-0.0186(0.385)$ & $-0.0193(0.393)$ \\
MATTR (50) & $-0.0956(0.0454)$ & $-0.254(3.46 \mathrm{e}-8)^{* * *}$ & $-0.158(1.13 \mathrm{e}-3){ }^{* *}$ \\
WordNet ambiguity (SD) - nouns & $-0.302(0.505)$ & $-1.28(4.29 \mathrm{e}-5)^{* * *}$ & $-0.974(3.70 \mathrm{e}-3)^{* *}$ \\
\hline
\end{tabular}

We report the $p$-values following a Tukey honest significant difference test with a $95 \%$ family-wise confidence level. ${ }^{* * *}$ denotes a $p$-value $<0.001,{ }^{* *}<0.01,{ }^{*}<0.05$.

used by participants in Session 1 display greater age-of-acquisition than those used in Session 3 . We find a few linguistic features that do no show any differences across sessions, such as word length and counts of function words.

4.2.3 Summary. We extracted various lexical, syntactic, and semantic features from transcripts of participant speech and looked for differences across Session, MMSE group, Participant, and 
Image Order. We found that some features vary across MMSE, with differences between the Mild and Severe group being the most prominent. Some features did not differ across Session but were influenced by MMSE (e.g., mean length of sentence).

\subsection{Qualitative Analysis}

At least two researchers were present at every session. During the recording sessions and the preand post-session questionnaires, researchers took notes of the participant's reactions. Following data collection, we read through the observation notes, the audio recordings and corresponding transcripts of the conversations. Here, we present some themes that emerged across all 19 participants with AD. In the provided examples, Hum denotes the human partner (Session 1), Woz the "Wizard-of-Oz" robot (Session 2), and autonomous Rob the robot (Session 3).

4.3.1 Likeability of the Robot. We observed mixed reactions with regards to robot likeability and eagerness to interact with the robot. Some participants were skeptical about using a robot and were noticeably less talkative in Sessions 2 and 3. For example, P3 (moderate-mild MMSE) was receptive to both open-ended and personal questions in the human-human session (e.g., Hum: "Ya. Do you like it by the water?," P3: "Yes," Hum: "It's really nice," P3: "We have a cottage!") but did not elaborate on answers to personal questions in Sessions 2 and 3, despite displaying no difficulties interacting with the robot. P3's answers were short during interactions with the robot. P2 (severe MMSE) indicated that she was eager to meet and interact with the robot during her presesion questionnaire. However, during sessions with the robot, she was quiet and unresponsive, only occasionally looking up at Ludwig. P16 (mild MMSE) was skeptical about the robot, calling him "just another toy" and did not wish to participate in Session 3, despite being receptive to the picture description task earlier.

In contrast, P12 (moderate MMSE) and P15 (severe MMSE) were initially nervous about meeting the robot but enjoyed their sessions with Ludwig, expressing gratitude at the end of the conversations (e.g., WoZ: "Are you happy?," P15: "Yes I'm happy, very happy,” WoZ: "What makes you happy?," P15: "Watching you!”).

4.3.2 Understanding and Intelligibility. Intelligibility and understanding were often issues with the robot, especially in the autonomous session. P9 and P13 struggled to understand the robot and would often turn to the researchers in the room whenever they did not understand the robot (e.g., Rob: "What is in the picture?" P13: "What is addition? Addition to uh what?"). P13 later noted in the post-session questionnaire that she "sometimes didn't understand" Ludwig. P16 and P14 also struggled to hear the robot, with P14's conversations suffering in Session 3 due to the autonomous robot's ability to reword utterances that needed to be repeated (e.g., Rob: "Do you like the beach?," P14: "Pardon?," Rob: "Do you like the beach?," P14: "Do I like the beach? Uh I can take it or leave it.").

However, despite some instances of misunderstanding, P12 claimed to enjoy her interactions with the robot and was looking forward to speaking with him again. P13 noted that the robot asked "reasonable questions" and P14 enjoyed her interactions with the robot (e.g., WoZ: "It was nice to meet you," P14: "Oh heavens. Thank you so much. I must say I'm very impressed with your performance."). Furthermore, in some cases, participants moved past the misunderstanding (e.g., Rob: "I am Ludwig," P12: "I didn't get what he said," Rob: "Can I ask you some questions on this picture?," Rob: “Certainly.”).

4.3.3 Intelligence of the Robot. Participants noted some limitations of the robot. P17 and P13 noted that the robot was was "not fast enough," which was especially a problem when long participant utterances slowed ASR. During the third session, P13 mentioned that she would have 
enjoyed the robot more if he moved, while P15 remarked that it "didn't look at me" when asked if she thought he understood what she was saying. Despite these difficulties, P13 and P15 acknowledged that the robot seemed friendly and intelligent, and P17 remarked that the robot was "good" and "soothing." P10's restless banging on the table significantly deteriorated the quality of speech recognition, yet she said talking to Ludwig was "fun."

Interestingly, P13 spoke in English with the robot, while she only responded in her native tongue to the human partner.

4.3.4 Eliciting Reactions. Overall, conversations were more successful with participants with higher MMSE scores. Participants like P2 (medium-mild) and P16 (mild) were talkative and correctly answered questions. However, keeping their attention of participants with lower MMSE scores was a challenge. For example, P4, P8, and P15 fell asleep during most of their sessions and were barely responsive. Since Ludwig was their first exposure to robots, we hoped that the presence of a new conversation partner could elicit interest. Surprisingly, P8 in fact spoke a few words in Session 3.

P9 (moderate MMSE) did not want to interact with her human partner and ended Session 1 early; however, the robot was able to capture her attention and she stayed for the entire duration with Ludwig.

Ludwig was not always successful in capturing the interest of participants. For example, P11 did not want to participate in the picture description task in Session 1 (e.g., H: "Can you look at the photo down here?, P11: "No," H: "No? Don't want to look at it?," P11: "No. I don't know anything") and did not answer questions in either of the robot sessions.

4.3.5 Correctness of Responses. Participants with higher MMSE scores typically described the contents of the pictures more fully than those with lower MMSE scores. Their responses to the questions had tended to be correct, regardless of whether their conversation partner was a human or Ludwig.

4.3.6 Summary. After going over conversation transcripts, observations from the sessions, and audio recordings, we synthesize our findings into the following themes: (1) Likeability of the robot was variable. (2) Understanding of the robot was an issue, especially in the autonomous session (i.e., Session 3) but had no effect on likeability. (3) Overall, participants thought the robot seemed intelligent, albeit slow to respond at times. (4) The robot's ability to elicit reactions was mixed. There were a few surprising instances where the robot was able to sustain the attention of a previously uncooperative participant. (5) Correctness of responses was more dependent on the participant's MMSE than on their conversation partner.

\section{DISCUSSION}

\subsection{Monitoring Cognitive Impairment through Language}

We report 15 linguistic features that differ across MMSE groups (Mild vs. Moderate vs. Severe), and none of the features identified in Table 10 show an effect of Image Order.

There are a few factors that show an effect of MMSE but not of Session (i.e., the word length and the mean length of sentence). These features are good candidates for linguistic markers that may be used for monitoring cognitive impairment in people with $\mathrm{AD}$, as they are sensitive to changes in MMSE but consistent throughout the three phrases of interaction (human, "Wizardof-Oz," and autonomous robot). This subset of features that differed across MMSE is less likely to be affected by further modifications and improvements to the conversational robot. To assess the feasibility of using robots for monitoring, assessment, and intervention of dementia, a higher level 
of clinical trial should be conducted. Nevertheless, this study offers promising results for using conversational robots in this population.

\subsection{Human and Robot}

In general, conversations were smoother and richer with the human conversational partner: Conversations were longer with a larger vocabulary, and participants were more "open" and engaged more with the human participant and engaged more in the repair process. However, we notice that the robot was able to capture the attention of participants that were seemingly bored or uninterested by conversation with a human partner.

These findings suggest that the barriers conversational robots face with individuals with dementia will not be in terms of their acceptance but in their fidelity. Most importantly, this includes the use of extra-linguistic information, such as gestures, facial expressions, and prosody, which were minimally used in our WoZ experiments. While past research has shown that robots are useful as a companion to older adults $[28,41,42]$, for a robot to be used effectively as a conversational companion, these limitations would need to be explored and addressed. However, given the success of the robot, especially with individuals having mild $\mathrm{AD}$, cognitive monitoring through conversation may be a feasible short-term goal.

\section{3 “Wizard-of-Oz" vs. Autonomy}

The "Wizard-of-Oz" setup allows us to evaluate participants' reactions to the robotic interface, whereas the autonomous version of the robot indicates the limits of existing software. Evidently, the autonomous version of the robot was not as flexible as its remotely controlled counterpart. It did not remember previous utterances or repeat or re-word misunderstood utterances.

The human conversation partner and the remotely controlled robot were able to respond to outof-domain vocabulary and flawed syntax, could sense whether a conversation should continue or end, and could interpret affect from facial expressions.

Our experiments highlight the need for variety and flexibility in dialogue; misunderstanding is unavoidable, and participants will have problems with intelligibility. Some recent work has used reinforcement learning to learn policies that can at least limit those instances [5].

The greatest barrier in producing a more flexible system is the limitations of ASR technology in natural environments for older adults. As this technology progresses, conversational systems can take advantage of improved models for language understanding and generation. However, handling communication breakdown will also be important in a successful system, and this will require further research.

\subsection{Limitations}

Alzheimer's disease is a degenerative disorder for which symptom expression can vary drastically from day to day. Since we performed our sessions over several months, we anticipated some cognitive decline between sessions and used MMSE scores as an evaluation of cognitive state. However, the MMSE scores cannot account for day-to-day symptomatic variation (e.g., good days and bad days). Although infeasible in this case, recording a larger number of conversations could mitigate this problem.

Although we designed our WoZ session to compare only the physical differences between a human and robot partner, technological limitations meant that the conversational abilities of the WoZ did not perfectly replicate those of the human partner. In the process of passing commands to the robot, the operator largely filtered out fragment and stall utterances (stuttering, rephrasing, etc.) and used more measured or thought-out utterances. Controlling the robot places extra cognitive burden on the operator. 
The autonomous robot also had several technical limitations. Primarily, the system is limited by the quality of ASR for older adults [46]. Through rule-based approaches and simple errorhandling, we produced a robust but inflexible system. The robot maintains no notion of dialogue context, ignores out-of-vocabulary words, and relies on keyword matching to guide dialogue flow. Furthermore, the autonomous robot does not have access to facial features, gestures, and prosody, which are important conversational cues, and is limited by the clarity of its synthetic voice, which is particularly important for interacting with older adults. Further work into building dialogue systems for older adults should aim to overcome these limitations or more fully analyze their impact on the quality of the conversions.

The design of the autonomous system was informed by our analysis of the WoZ conversations. Unfortunately, this did not permit counterbalancing of the order of the WoZ and autonomous conditions; always performing the WoZ condition first may have biased the participants toward expectations of the autonomous system beyond its capabilities.

Our linguistic analysis for cognitive monitoring has followed the work of Fraser et al. [11] and Roark et al. [32] and is restricted to examining features that can be automatically extracted from transcriptions of conversations. Although miscommunication could be a useful feature in cognitive monitoring, it has not been included in this analysis, because TSR variables were manually annotated. However, these features would be useful in assessing dementia. Similarly, acoustic features would also likely be useful in cognitive monitoring, but were not included in our analysis.

Most notably, the limited number of participants in this study limits our ability to generalize our findings. The MMSE scores of some participants were very low, causing them to have trouble interacting with the device. While we were able to derive some linguistic measures and qualitative observations from these participants, further research will be required in the area of conversational robotics for individuals with dementia to substantiate our results.

Our pilot highlighted several limitations in the autonomous version of the robot, including inflexible dialogue, an inability to adapt to new situations, and limitations of ASR technology for this atypical population. Future work should focus on the tradeoffs between autonomous and semiautonomous approaches.

\section{CONCLUSIONS}

As the population ages, technological interventions will be needed to offset the increased burden placed on caregivers. In this pilot study with the robot Ludwig, we explore conversational agents for cognitive assessment by engaging with individuals with dementia in picture description dialogues. Overall, we observe mixed reactions to the robot, although many participants reflected positively on their interactions. The most successful of these, and the richest conversations, occurred with individuals with milder cognitive impairment. Robotic agents are likely to be most appropriate for this group.

Decreased intelligibility of the robot's synthetic voice (and the lack of flexible prosodic, gestural, and facial cues) may have contributed to the participants being less responsive to a "Wizard-of-Oz" robot. This problem was intensified with the autonomous robot that did not have the ability to identify and recover from communication breakdowns. Given that the burden of repair is placed on the partners of individuals with dementia [30], it is important that conversational agents are able to participate in the repair process. By making the data collected with Ludwig available, our goal is to foster research into conversational systems that recover from and repair breakdowns in communication when they occur. With Ludwig, we demonstrate the type of automatic linguistic analysis that we envision being used in longitudinal monitoring and assessment of dementia. For this kind of promising analysis to become a practical clinical tool-and for flexible conversational agents to become robust enough to be used in practice-improvements will be required in 
automatic speech recognition to target the voices of older adults with speech impairments. We hope such technology can be used to help inform the development of treatment plans and the promotion of independent living of individuals with dementia.

\section{APPENDICES}

\section{A USABILITY QUESTIONNAIRES}

\section{A.1 Pre-session Questionnaire}

The pre-session questionnaire is administered before interactions with the robot.

Table 13. Questions Used in the Pre-session Questionnaire

\begin{tabular}{l}
\hline Technology use and familiarity \\
\hline Have you used a tablet computer before? \\
How comfortable are you with a tablet? \\
\hline Robot interaction \\
\hline It would be good to have a robot help \\
I am nervous about interacting \\
Using a robot would be exciting \\
\hline
\end{tabular}

\section{A.2 Post-session Questionnaire}

The post-session questionnaire is administered after interactions with the robot.

Table 14. Questions Used in the Post-session Questionnaire

Using the robot was stressful.
I would like to speak with the robot again.
What do you think about the robot?
What do you like about the robot?
What do you dislike about the robot?
I could understand the robot.
Talking with the robot felt natural.
The robot understood what I said to it.
The robot let me know when it did not understand me.
How did you feel when you were talking with the robot?
I think the robot is nice/friendly.
The robot seems intelligent.
In what ways should the robot be improved?
Do you have any other comments about the study?

\section{B LINGUISTIC FEATURES}

\section{B.1 Syntactic Features}

We parse the transcripts and then compute various syntactic features, including phrase type length and proportion. The features in Table 15 were identified in our linguistic analysis (Section 3.4.2). 
Table 15. Description of Syntactic Features Identified by Linguistic Analysis

\begin{tabular}{|cl|}
\hline \multicolumn{1}{|c|}{ Feature } & \multicolumn{1}{c|}{ Description } \\
\hline Average PP length & $\begin{array}{l}\text { Average length of the preposition phrase (PP) grammatical constituent, } \\
\text { as defined by the total number of words in PP divided by number of } \\
\text { phrases of type PP. } \\
\text { Average VP length } \\
\text { Average length of the verb phrase (VP) grammatical constituent, as } \\
\text { defined by the total number of words in VP divided by number of } \\
\text { phrases of type VP. } \\
\text { PP type proportion } \\
\text { Length of each preposition phrase (PP) divided by total number of } \\
\text { words in transcript. }\end{array}$ \\
\hline
\end{tabular}

\section{B.2 Lexical Features}

We compute lexical features related to the number of syllables and number of characters in words, as well as various measures of vocabulary richness. The features in Table 16 were identified in our linguistic analysis (Section 3.4.2).

Table 16. Description of Lexical Features Identified by Linguistic Analysis

\begin{tabular}{|c|c|}
\hline Feature & Description \\
\hline Word length & The mean number of characters per word. \\
\hline Age-of-acquisition - nouns & $\begin{array}{l}\text { Mean values of age-of-acquisition (i.e., the age at which a person learned } \\
\text { a word) for all nouns, based on the Bristol [37] and Gilhoolie-Logie [14] } \\
\text { ratings. }\end{array}$ \\
\hline MATTR (40) & $\begin{array}{l}\text { Moving average type-token-ratio: Shift a window of size } k=40 \text { over the } \\
\text { transcript and compute the type-token-ratio over each, then average over } \\
\text { all windows [7]. }\end{array}$ \\
\hline MATTR (20) & $\begin{array}{l}\text { Moving average type-token-ratio: Shift a window of size } k=20 \text { over the } \\
\text { transcript and compute the type-token-ratio over each, then average over } \\
\text { all windows [7]. }\end{array}$ \\
\hline Familiarity - nouns & $\begin{array}{l}\text { Mean values of age-of-acquisition (i.e., how often a word is used, seen or } \\
\text { heard) for all nouns, based on the Bristol [37] and Gilhoolie-Logie [14] } \\
\text { ratings. }\end{array}$ \\
\hline MATTR (30) & $\begin{array}{l}\text { Moving average type-token-ratio: Shift a window of size } k=30 \text { over the } \\
\text { transcript and compute the type-token-ratio over each, then average over } \\
\text { all windows [7]. }\end{array}$ \\
\hline Function words & $\begin{array}{l}\text { Total number of function words (i.e., a word whose purpose is more to } \\
\text { signal grammatical relationship than the lexical meaning of a sentence) } \\
\text { normalized by total number of words in transcript. }\end{array}$ \\
\hline Mean length of sentence & Mean number of words per sentence. \\
\hline MATTR (50) & $\begin{array}{l}\text { Moving average type-token-ratio: Shift a window of size } k=50 \text { over the } \\
\text { transcript and compute the type-token-ratio over each, then average over } \\
\text { all windows [7]. }\end{array}$ \\
\hline
\end{tabular}

\section{B.3 Semantic Features}

Semantic features were derived from WordNet [26]. The top semantic features identified by linguistic analysis (Section 3.4.2) are described in Table 17. 
Table 17. Description of Semantic Features Identified by Linguistic Analysis

\begin{tabular}{|cl|}
\hline Feature & \multicolumn{1}{c|}{ Description } \\
\hline Min. WordNet depth (SD) - nouns & $\begin{array}{l}\text { The standard deviation of the minimum WordNet depth for all } \\
\text { nouns. } \\
\text { Max. WordNet depth (SD) - nouns }\end{array}$ \\
$\begin{array}{l}\text { The standard deviation of the maximum WordNet depth for all } \\
\text { nouns. } \\
\text { WordNet ambiguity - nouns }\end{array}$ & The average number of senses in WordNet for all nouns. \\
\hline
\end{tabular}

\section{ACKNOWLEDGMENTS}

We thank Selvana Morcos, Olivia Rogoza, and Hannah Der for providing transcripts of the conversations and annotations. We thank the reviewers for their feedback.

\section{REFERENCES}

[1] Mohamed Abdalla, Frank Rudzicz, and Graeme Hirst. 2017. Rhetorical structure and alzheimer's disease. Aphasiology 32, 1 (2017), 1-20. DOI : https://doi.org/10.1080/02687038.2017.1355439

[2] Jörgen Ahlberg. 2001. CANDIDE-3 - An Updated Parameterised Face. Technical Report.

[3] Clive Ballard, Serge Gauthier, Anne Corbett, Carol Brayne, Dag Aarsland, and Emma Jones. 2011. Alzheimer's disease. Lancet 377, 9770 (Mar. 2011), 1019-31. DOI : https://doi.org/10.1016/S0140-6736(10)61349-9

[4] Alistair Burns and Steve Iliffe. 2009. Alzheimer's disease. Br. Med. J. 338, b158 (2009). DOI : https://doi.org/10.1136/ bmj.b158 arXiv:http://www.bmj.com/content/338/bmj.b158

[5] Hamidreza Chinaei, Leila Chan Currie, Andrew Danks, Hubert Lin, Tejas Mehta, and Frank Rudzicz. 2017. Identifying and avoiding confusion in dialogue with people with alzheimer's disease. Comput. Ling. 43, 2 (Mar. 2017), 1-42. DOI : https://doi.org/10.1162/COLI_a_00290

[6] Cathleen M. Connell, Mary R. Janevic, and Mary P. Gallant. 2001. The costs of caring: Impact of dementia on family caregivers. f. Geriatr. Psychiat. Neurol. 14, 4 (Dec. 2001), 179-187. DOI : https://doi.org/10.1177/089198870101400403

[7] Michael A. Covington and Joe D. McFall. 2010. Cutting the gordian knot: The moving-average type-token ratio (MATTR). F. Quant. Ling. 17, 2 (May 2010), 94-100. DOI : https://doi.org/10.1080/09296171003643098

[8] Juliana Onofre De Lira, Karin Zazo Ortiz, Aline Carvalho Campanha, Paulo Henrique Ferreira Bertolucci, and Thaís Soares Cianciarullo Minett. 2011. Microlinguistic aspects of the oral narrative in patients with alzheimer's disease. Int. Psychogeriatr. 23, 03 (Apr. 2011), 404-412. DOI : https://doi.org/10.1017/S1041610210001092

[9] Marshal F. Folstein, Susan E. Folstein, and Paul R. McHugh. 1975. "Mini-mental state". A practical method for grading the cognitive state of patients for the clinician. F. Psychiatr. Res. 12, 3 (1975), 189-198.

[10] Katrina E. Forbes-McKay and Annalena Venneri. 2005. Detecting subtle spontaneous language decline in early alzheimer's disease with a picture description task. Neurol. Sci. 26, 4 (Oct. 2005), 243-254. DOI: https://doi.org/10. 1007/s10072-005-0467-9

[11] Kathleen C. Fraser, Jed A. Meltzer, and Frank Rudzicz. 2015. Linguistic features identify Alzheimer's disease in narrative speech. F. Alzheim. Dis. 49, 2 (2015), 407-422. DOI : https://doi.org/10.3233/JAD-150520

[12] Dennis Getsios, Steve Blume, Khajak J. Ishak, Grant Maclaine, and Luis Hernández. 2012. An economic evaluation of early assessment for alzheimer's disease in the united kingdom. Alzheim. Dement. 8, 1 (Jan. 2012), 22-30. DOI: https://doi.org/10.1016/j.jalz.2010.07.001

[13] Elaine Giles, Karalyn Patterson, and John R. Hodges. 1996. Performance on the boston cookie theft picture description task in patients with early dementia of the alzheimer's type: Missing information. Aphasiology 10, 4 (May 1996), 395408. DOI : https://doi.org/10.1080/02687039608248419

[14] Ken J. Gilhooly and Robert H. Logie. 1980. Age-of-acquisition, imagery, concreteness, familiarity, and ambiguity measures for 1,944 words. Behav. Res. Methods Instrum. 12, 4 (Jul. 1980), 395-427. DOI : https://doi.org/10.3758/BF03201693

[15] Liesi E. Hebert, Jennifer Weuve, Paul A. Scherr, and Denis A. Evans. 2013. Alzheimer disease in the united states (2010-2050) estimated using the 2010 census. Neurology 80, 19 (May 2013), 1778-83. DOI : https://doi.org/10.1212/WNL. 0b013e31828726f5

[16] Marcel Heerink, Ben Kröse, Vanessa Evers, and Bob J. Wielinga. 2007. Observing conversational expressiveness of elderly users interacting with a robot and screen agent. In Proceedings of the 2007 IEEE 10th International Conference on Rehabilitation Robotics. IEEE, 751-756. DOI : https://doi.org/10.1109/ICORR.2007.4428509

[17] Marcel Heerink, Ben Kröse, Bob J. Wielinga, Vanessa Evers, et al. 2006. Studying the acceptance of a robotic agent by elderly users. Int. f. Assist. Robot. Mechatron. 7, 3 (2006), 33-43. 
[18] Julie D. Henry, John R. Crawford, and Louise H. Phillips. 2004. Verbal fluency performance in dementia of the Alzheimer's type: A meta-analysis. Neuropsychologia 42, 9 (Jan. 2004), 1212-1222. DOI: https://doi.org/10.1016/j. neuropsychologia.2004.02.001

[19] William Jarrold, Bart Peintner, David Wilkins, Dimitra Vergryi, Colleen Richey, Maria Luisa Gorno-Tempini, and Jennifer Ogar. 2014. Aided diagnosis of dementia type through computer-based analysis of spontaneous speech. In Proceedings of the Workshop on Computational Linguistics and Clinical Psychology: From Linguistic Signal to Clinical Reality. 27-37. DOI: https://doi.org/10.3115/v1/W14-3204

[20] H. S. Kirshner, W. G. Webb, and M. P. Kelly. 1984. The naming disorder of dementia.Neuropsychologia 22, 1 (1984), 23-30.

[21] Majid Komeili, Chloe Pou-Prom, Daniyal Liaqat, Kathleen C. Fraser, Maria Yancheva, and Frank Rudzicz. 2019. Talk2Me: Automated linguistic data collection for personal assessment. PLoS ONE 14, 3 (2019).

[22] Catherine Lai, Jean Carletta, and Steve Renals. 2013. Modelling participant affect in meetings with turn-taking features. In Proceedings of the Workshop on Affective Social Speech Signals (WASSS'13).

[23] Hee Rin Lee and Laurel D. Riek. 2018. Reframing assistive robots to promote successful aging. ACM Trans. Hum.-Robot Interact. 7, 1, Article 11 (May 2018), 23 pages. DOI : https://doi.org/10.1145/3203303

[24] Francisco Martín, Carlos E. Agüero, José M. Cañas, Meritxell Valenti, and Pablo Martínez-Martín. 2013. Robotherapy with dementia patients. Int. f. Adv. Robot. Syst. 10, 1 (2013), 10. DOI:https://doi.org/10.5772/54765 arXiv:http://dx.doi.org/10.5772/54765

[25] Guy M. McKhann, David S. Knopman, Howard Chertkow, Bradley T. Hyman, Clifford R. Jack, Claudia H. Kawas, William E. Klunk, Walter J. Koroshetz, Jennifer J. Manly, Richard Mayeux, Richard C. Mohs, John C. Morris, Martin N. Rossor, Philip Scheltens, Maria C. Carrillo, Bill Thies, Sandra Weintraub, and Creighton H. Phelps. 2011. The diagnosis of dementia due to alzheimer's disease: Recommendations from the national institute on aging-alzheimer's association workgroups on diagnostic guidelines for alzheimer's disease. Alzheim. Dement. 7, 3 (2011), 263-269. DOI : https://doi.org/10.1016/j.jalz.2011.03.005

[26] George A. Miller. 1995. WordNet: A lexical database for english. Commun. ACM 38, 11 (Nov. 1995), 39-41. DOI: https://doi.org/10.1145/219717.219748

[27] Elaine Mordoch, Angela Osterreicher, Lorna Guse, Kerstin Roger, and Genevieve Thompson. 2013. Use of social commitment robots in the care of elderly people with dementia: A literature review. Maturitas 74, 1 (2013), 14-20. DOI : https://doi.org/10.1016/j.maturitas.2012.10.015

[28] Wendy Moyle, Marie Cooke, Elizabeth Beattie, Cindy Jones, Barbara Klein, Glenda Cook, and Chrystal Gray. 2013. Exploring the effect of companion robots on emotional expression in older adults with dementia: A pilot randomized controlled trial. F. Gerontol. Nurs. 39, 5 (May 2013), 46-53. DOI : https://doi.org/10.3928/00989134-20130313-03

[29] Kimberly D. Mueller, Bruce Hermann, Jonilda Mecollari, and Lyn S. Turkstra. 2018. Connected speech and language in mild cognitive impairment and alzheimer's disease: A review of picture description tasks. 7. Clin. Exp. Neuropsychol. 40, 9 (2018), 1-23. DOI : https://doi.org/10.1080/13803395.2018.1446513

[30] Joseph B. Orange, Rosemary B. Lubinski, and D. Jeffery Higginbotham. 1996. Conversational repair by individuals with dementia of the alzheimer's type. F. Speech Hear. Res. 39, 4 (Aug. 1996), 881-95.

[31] Bart Peintner, William Jarrold, Dimitra Vergyri, Colleen Richey, Maria Luisa Gorno Tempini, and Jennifer Ogar. 2008. Learning diagnostic models using speech and language measures. In Proceedings of the 2008 30th Annual International Conference of the IEEE Engineering in Medicine and Biology Society, Vol. 2008. IEEE, 4648-4651. DOI : https://doi.org/ 10.1109/IEMBS.2008.4650249

[32] Brian Roark, Margaret Mitchell, John-Paul Hosom, Kristy Hollingshead, and Jeffrey Kaye. 2011. Spoken language derived measures for detecting mild cognitive impairment. IEEE Trans. Audio Speech Lang. Process. 19, 7 (Sep. 2011), 2081-2090. DOI : https://doi.org/10.1109/TASL.2011.2112351

[33] Frank Rudzicz, Rosalie Wang, Momotaz Begum, and Alex Mihailidis. 2015. Speech interaction with personal assistive robots supporting aging at home for individuals with alzheimer's disease. ACM Trans. Access. Comput. 7, 2 (2015), $1-22$.

[34] Selma Sabanovic, Casey C. Bennett, Wan-Ling Chang, and Lesa Huber. 2013. PARO robot affects diverse interaction modalities in group sensory therapy for older adults with dementia. In Proceedings of the 2013 IEEE 13th International Conference on Rehabilitation Robotics (ICORR'13). IEEE, 1-6. DOI : https://doi.org/10.1109/ICORR.2013.6650427

[35] Yoichi Sakai, Yuuko Nonaka, Kiyoshi Yasuda, and Yukiko I. Nakano. 2012. Listener agent for elderly people with dementia. In Proceedings of the 7th Annual ACM/IEEE International Conference on Human-Robot Interaction (HRI'12). ACM Press, New York, NY, 199. DOI : https://doi.org/10.1145/2157689.2157754

[36] Takanori Shibata and Kazuyoshi Wada. 2011. Robot therapy: A new approach for mental healthcare of the elderly a mini-review. Gerontology 57, 4 (2011), 378-86. DOI : https://doi.org/10.1159/000319015

[37] Hans Stadthagen-Gonzalez and Colin J. Davis. 2006. The bristol norms for age of acquisition, imageability, and familiarity. Behav. Res. Methods 38, 4 (Nov. 2006), 598-605. DOI : https://doi.org/10.3758/BF03193891 
[38] Ruth M. Tappen, Christine L. Williams, Charlotte Barry, and Donna Disesa. 2002. Conversation intervention with alzheimer's patients: Increasing the relevance of communication. Clin. Gerontol. 24, 3-4 (2002), 63-75. DOI : https://doi.org/10.1300/J018v24n03_06

[39] Adriana Tapus, Juan Fasola, and Maja J. Matarić. 2008. Cognitive assistance and physical therapy for dementia patients. In ICRA Workshop on Social Interaction with Intelligent Indoor Robots.

[40] Rik van den Brule, Gijsbert Bijlstra, Ron Dotsch, Pim Haselager, and Daniel H. J. Wigboldus. 2016. Warning signals for poor performance improve human-robot interaction. J. Hum.-Robot Interact. 5, 2 (2016), 69-89.

[41] Kazuyosh Wada and Takanori Shibata. 2007. Living with seal robots-Its sociopsychological and physiological influences on the elderly at a care house. IEEE Trans. Robot. 23, 5 (Oct. 2007), 972-980. DOI : https://doi.org/10.1109/TRO. 2007.906261

[42] Kazuyosh Wada, Takanori Shibata, Toshimitsu Musha, and Shin Kimura. 2008. Robot therapy for elders affected by dementia. IEEE Eng. Med. Biol. Mag. 27, 4 (Jul. 2008), 53-60. DOI : https://doi.org/10.1109/MEMB.2008.919496

[43] Caroline M. Watson, Helen J. Chenery, and Michelle S. Carter. 1999. An analysis of trouble and repair in the natural conversations of people with dementia of the alzheimer's type. Aphasiology 13, 3 (Mar. 1999), 195-218. DOI : https://doi.org/10.1080/026870399402181

[44] Bengt Winblad, Philippe Amouyel, Sandrine Andrieu, Clive Ballard, Carol Brayne, Henry Brodaty, Angel CedazoMinguez, Bruno Dubois, David Edvardsson, Howard Feldman, Laura Fratiglioni, Giovanni B. Frisoni, Serge Gauthier, Jean Georges, Caroline Graff, Khalid Iqbal, Frank Jessen, Gunilla Johansson, Linus Jönsson, Miia Kivipelto, Martin Knapp, Francesca Mangialasche, René Melis, Agneta Nordberg, Marcel Olde Rikkert, Chengxuan Qiu, Thomas P. Sakmar, Philip Scheltens, Lon S. Schneider, Reisa Sperling, Lars O. Tjernberg, Gunhild Waldemar, Anders Wimo, and Henrik Zetterberg. 2016. Defeating alzheimer's disease and other dementias: A priority for european science and society. Lancet Neurol. 15, 5 (Apr. 2016), 455-532. DOI : https://doi.org/10.1016/S1474-4422(16)00062-4

[45] Maria Yancheva, Kathleen C. Fraser, and Frank Rudzicz. 2015. Using linguistic features longitudinally to predict clinical scores for alzheimer's disease and related dementias. In Proceedings of the 6th Workshop on Speech and Language Processing for Assistive Technologies (SLPAT'15). 134. DOI : https://doi.org/10.1016/j.bandl.2008.08.002

[46] Victoria Young and Alex Mihailidis. 2010. Difficulties in automatic speech recognition of dysarthric speakers and implications for speech-based applications used by the elderly: A literature review. Assist. Technol. 22, 2 (2010), 99112 .

[47] Luke Zhou, Kathleen C. Fraser, and Frank Rudzicz. 2016. Speech recognition in alzheimer's disease and in its assessment. In Proceedings of the 17th Annual Meeting of the International Speech Communication Association (Interspeech'16) 1948-1952. DOI : https://doi.org/10.21437/Interspeech.2016-1228

Received April 2019; revised November 2019; accepted January 2020 